\title{
ARITHMETIC HYPERBOLIC REFLECTION GROUPS
}

\author{
MIKHAIL BELOLIPETSKY \\ Dedicated to Ernest Borisovich Vinberg
}

Abstract. A hyperbolic reflection group is a discrete group generated by reflections in the faces of an $n$-dimensional hyperbolic polyhedron. This survey article is dedicated to the study of arithmetic hyperbolic reflection groups with an emphasis on the results that were obtained in the last ten years and on the open problems.

\section{Contents}

1. Introduction

2. Quadratic forms and arithmetic reflection groups

3. The work of Nikulin

4. Spectral method and finiteness theorems

5. Effective results obtained by the spectral method

6. Classification results in small dimensions

7. Examples

8. Reflective modular forms

9. More about the structure of the reflective quotient

10. Open problems

Acknowledgments

About the author

References

\section{INTRODUCTION}

Consider a finite volume polyhedron $\mathrm{P}$ in the $n$-dimensional hyperbolic space $\mathbb{H}^{n}$. It may occur that if we act on $\mathrm{P}$ by the hyperbolic reflections in its sides, the images would cover the whole space $\mathbb{H}^{n}$ and would not overlap with each other. In this case we say that the transformations form a hyperbolic reflection group $\Gamma$ and that $\mathrm{P}$ is its fundamental polyhedron, also known as the Coxeter polyhedron of $\Gamma$. We can give an analogous definition of the spherical and euclidean reflection groups, the classes of which are well understood after the work of Coxeter [Cox34]. What kind of properties characterize hyperbolic Coxeter polyhedra? For example, we have to assume that all the dihedral angles of $\mathrm{P}$ are of the form $\frac{\pi}{m}$ for some $m \in\{2,3, \ldots, \infty\}$ because otherwise some images of $\mathrm{P}$ over $\Gamma$ would overlap. It

Received by the editors September 21, 2015.

2010 Mathematics Subject Classification. Primary 22E40; Secondary 11F06, 11H56, 20H15, $51 \mathrm{~F} 15$. 
appears that under some natural assumptions one can prove a general finiteness theorem for the possible types of hyperbolic Coxeter polyhedra in all dimensions. In this paper we shall give an overview of the related methods, results, and open problems.

Reflection groups are ubiquitous in mathematics: they appear in group theory, Riemannian geometry, number theory, algebraic geometry, representation theory, singularity theory, low-dimensional topology, and other fields. A vivid description of the history of spherical and euclidean reflection groups can be found in the Note Historique of Bourbaki's volume [Bou68. The study of the spherical ones goes back to the mid-nineteenth century geometrical investigations of Möbius and Schläfli. It then continued in the work of Killing, Cartan, and Weyl on Lie theory. In a remarkable paper published in 1934 [Cox34, Coxeter gave a complete classification of irreducible spherical and euclidean reflection groups. Hyperbolic reflection groups in dimension 2 were described by Poincaré and Dyck already in the 1880s Poi82, Dyc82; they then played a prominent role in the work of Klein and Poincaré on discrete groups of isometries of the hyperbolic plane. Much later, in 1970, Andreev proved an analogous result for the hyperbolic three-space giving a classification of convex finite volume polyhedra in $\mathbb{H}^{3}$ And70a, And70b. Later on Andreev's theorem played a fundamental role in Thurston's work on geometrization of three-dimensional manifolds. The history and results about reflection groups in algebraic geometry are thoroughly discussed in Dolgachev's survey paper Dol08] and his lecture notes Dol15, the latter giving more details and being more focused on hyperbolic groups. Concluding this very brief overview, let us mention that many connections between reflection groups and group theory, combinatorics, and geometry can be found in the book by Conway and Sloane CS99.

Let us recall some well known examples of hyperbolic reflection groups. Let the dimension $n=2$, and consider geodesic triangles $\mathrm{P}_{1}$ and $\mathrm{P}_{2}$ in the hyperbolic plane with the angles $\frac{\pi}{2}, \frac{\pi}{3}, \frac{\pi}{7}$ and $\frac{\pi}{2}, \frac{\pi}{3}, 0=\frac{\pi}{\infty}$, respectively. The corresponding reflection groups $\Gamma_{1}$ and $\Gamma_{2}$ are discrete subgroups of the group of isometries of the hyperbolic plane. The first of them is known as the Hurwitz triangle group. It is ultimately related with the Klein quartic surface $\mathcal{X}$, the corresponding tiling of the fundamental domain of $\mathcal{X}$ on the hyperbolic plane which is shown in Figure 1 appeared in Klein's 1879 paper Kle79. This group has many remarkable properties, and a fascinating discussion of the related topics can be found in a book Lev99. The second group has an unbounded fundamental polyhedron whose hyperbolic area is finite (and $=\frac{\pi}{6}$ ), and it is isomorphic to the extended modular group $\operatorname{PGL}(2, \mathbb{Z})$. Changing to the dimension $n=3$, we encounter the right-angled dodecahedron whose corresponding tiling of the hyperbolic three-space as seen from within is represented in Figure 2. This image was produced by the Geometry Center at the University of Minnesota in the late 1990s, and among other places it appeared in the video "Not Knot" available at the Geometry Center homepage [GC] and on the cover of the published edition of Thurston's celebrated lecture notes [Thu97. The theories of Klein-Poincaré and Thurston were developed for studying much more general classes of discrete groups of isometries, but in both cases hyperbolic reflection groups provided a source of important motivating examples.

Similar to the way that the polygon in Figure 1 is tiled by triangles, the higherdimensional hyperbolic polyhedra may admit decompositions into smaller parts. A 


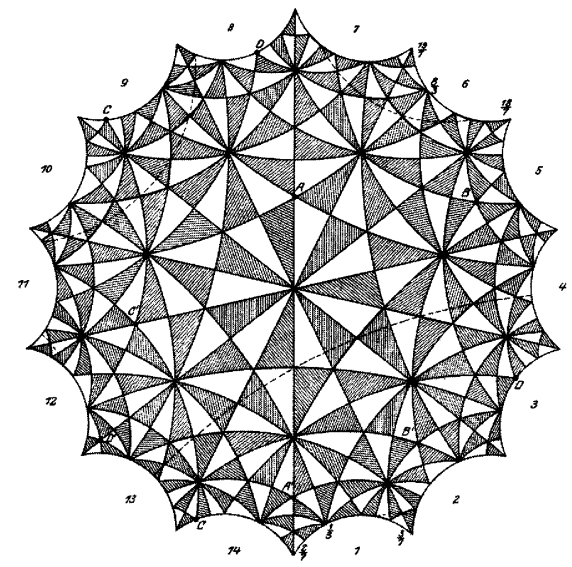

Figure 1. A tiling of a domain on the hyperbolic plane by the $(2,3,7)$-triangles (image taken from Klein's original article Kle79 and used with permission).

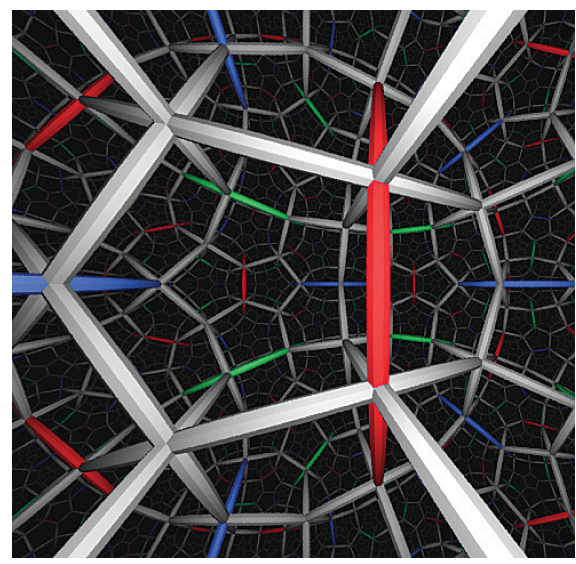

Figure 2. A tiling of the hyperbolic three-space by the right-angled dodecahedra (image courtesy of Geometry Center, University of Minnesota).

nice computer visualization of some three-dimensional tilings of this kind including two different decompositions of the right-angled dodecahedron is presented in ACMR09. In dimensions higher than 3 it is much harder to draw polyhedra and tilings but we can still study them using different methods. Moreover, the combinatorial and geometrical structure of the fundamental polyhedron of a hyperbolic reflection group in dimension $n \geq 3$ is uniquely determined by its Coxeter diagram (see Section 21). Throughout this paper we shall study the groups acting in spaces of arbitrary dimensions.

In what follows we are going to restrict our attention to arithmetic hyperbolic reflection groups. The definition of arithmeticity will be given in the next section; here we just note that all the examples considered above are arithmetic. Some results about the general class of hyperbolic reflection groups were discussed in the survey paper by Vinberg [Vin85]. For the methods that will feature in the present survey, arithmeticity plays an essential role.

We now recall a fundamental theorem of Vinberg [Vin81,Vin84a], which is well known and at the same time remains surprising:

Theorem 1.1. There are no arithmetic hyperbolic reflection groups in dimensions $n \geq 30$.

One may suspect that the yet to be defined notion of arithmeticity is crucial here but the conjecture is that it is not the case:

Conjecture 1.2. Theorem 1.1 is true without the arithmeticity assumption.

In fact, in his paper Vinberg proved two different theorems, one of which says that there are no cocompact hyperbolic reflection groups in dimensions $n \geq 30$, and the other that there are no non-cocompact arithmetic hyperbolic reflection groups in these dimensions. The coincidence of the dimensions in the two theorems is accidental. Later on Prokhorov proved that there are no non-cocompact reflection groups in dimensions $n \geq 996$ thus confirming the conjecture for sufficiently large 
$n$ Pro86]. There is no reason to expect any of these bounds for the dimension to be sharp. In Ess96, Esselmann was able to show that the dimensions of noncocompact arithmetic reflection groups satisfy $n \leq 21, n \neq 20$. This result is sharp because of the known examples due to Vinberg and Borcherds (see Section 7 ). Non-arithmetic hyperbolic reflection groups in dimensions larger than two were first constructed by Makarov and Vinberg in the 1960s. The examples of such groups are currently known for dimensions $n \leq 12, n=14$, 18 Vin14.

In connection with Theorem 1.1 let us mention the following more general question communicated to me by Anton Petrunin:

Question. Do there exist hyperbolic lattices (i.e., discrete cofinite isometry groups) generated by elements of finite order in spaces of large dimension?

The expected answer to this question is "No", but it is far from settled. A related discussion can be found on the MathOverflow page [MOv12]. There is a hope that the methods considered in this survey may be applied for attacking this problem for arithmetic lattices, but up to now our attempts to do it were not successful. We shall come back to this question in Section 10, which is dedicated to open problems.

Another principal question is what happens in the dimensions for which there do exist arithmetic reflection groups - how do we construct the examples of such groups and is it possible to classify all of them? These questions will be in the focus of the discussion in our survey.

The content of the paper is as follows. In Section 2 we recall the definition of arithmetic hyperbolic reflection groups and some well known results about them. Section 3 contains a brief discussion of the work of Nikulin on finiteness results for hyperbolic reflection groups. In the next section we introduce what we call the spectral method and discuss the finiteness theorems obtained by this method. Some effective results that are obtained by the spectral method are discussed in Section 5. The following Section 6] is dedicated to what is currently known about classification of arithmetic hyperbolic reflection groups. Section 7 presents a collection of examples with an emphasis on those that were discovered after Vinberg's 1985 survey paper was published. Some particularly interesting examples were obtained by Borcherds using modular forms, and we dedicate the next section to a discussion of the reflective modular forms. In Section 9 we consider the reflective quotients, in particular, the so-called quasi-reflective groups and also non-reflective groups. Finally, in the last section we discuss open problems.

Before starting the paper, let us cite three important articles that provide an overview of the subject from different perspectives. These are the papers by Nikulin Nik81a, Vinberg Vin85, and Dolgachev Dol08. The related results were also presented in ICM talks by Vinberg [Vin84b] and Nikulin [Nik87]. When it is possible, we shall try to minimize the overlap with these papers and focus our attention on the results that were obtained in the last ten years.

\section{QuAdratic FORMS AND ARITHMETIC REFLECTION GROUPS}

Consider an $(n+1)$-dimensional vector space $\mathbb{E}^{n, 1}$ with the inner product defined by a quadratic form $f$ of signature $(n, 1)$. Let

$$
\left\{v \in \mathbb{E}^{n, 1} \mid(v, v)<0\right\}=\mathfrak{C} \cup(-\mathfrak{C}),
$$

where $\mathfrak{C}$ is an open convex cone. In the vector model, the hyperbolic space $\mathbb{H}^{n}$ is identified with the set of rays through the origin in $\mathfrak{C}$, or $\mathfrak{C} / \mathbb{R}^{+}$, so that the 
isometries of $\mathbb{H}^{n}$ are the orthogonal transformations of $\mathbb{E}^{n, 1}$. We refer to AVS93. and Rat06] for a detailed study of the properties of the vector model.

Let $k$ be a totally real number field with the ring of integers $\mathfrak{o}_{k}$, and let $f$ be a quadratic form of signature $(n, 1)$ defined over $k$ and such that for every non-identity embedding $\sigma: k \rightarrow \mathbb{R}$, the form $f^{\sigma}$ is positive definite. The group $\Gamma=\mathrm{O}_{0}\left(f, \mathfrak{o}_{k}\right)$ of the integral automorphisms of $f$ is a discrete subgroup of $H=\mathrm{O}_{0}(n, 1)$, which is the full group of isometries of the hyperbolic $n$-space $\mathbb{H}^{n}$ (the group $\mathrm{O}_{0}(n, 1)$ is the subgroup of the orthogonal group $\mathrm{O}(n, 1)$ that preserves the cone $\mathfrak{C})$. The discreteness of $\Gamma$ follows from the discreteness of $\mathfrak{o}_{k}$ in $\mathbb{R}^{[k: \mathbb{Q}]}$ and compactness of $\mathrm{O}_{0}\left(f^{\sigma}\right)$ for $\sigma \neq \sigma_{i d}$. Using reduction theory, it can be shown that the covolume of $\Gamma$ in $H$ is finite - this is a special case of the fundamental theorem of Borel and HarishChandra BHC62] (we refer to the book VGS88 for an accessible exposition of the main ideas of the proof). Discrete subgroups of finite covolume are called lattices. The groups $\Gamma$ obtained in this way and subgroups of $H$ which are commensurable with them are called arithmetic lattices of the simplest type. The field $k$ is called the field of definition of $\Gamma$ (and subgroups commensurable with it). We shall also apply this terminology to the corresponding quotient orbifolds $\mathbb{H}^{n} / \Gamma$.

There are compact and finite volume non-compact arithmetic quotient spaces. A theorem known as the Godement's compactness criterion implies that $\Gamma$ is noncocompact if and only if $\mathrm{O}_{0}(f, k)$ has a non-trivial unipotent element [BHC62] (see also [VGS88, Chapter 3, Section 3.3]). This is equivalent to the condition that $k=\mathbb{Q}$ and the form $f$ is isotropic. The Hasse-Minkowski theorem implies that for $k=\mathbb{Q}$ and $n \geq 4$ the latter condition automatically holds. Therefore, for $n \geq 4$ the quotient $\mathbb{H}^{n} / \Gamma$ is non-compact if and only if $\Gamma$ is defined over the rationals. For $n=2$ and 3 , the non-cocompact subgroups are still defined over $\mathbb{Q}$ but there also exist cocompact arithmetic subgroups with the same field of definition.

In general, arithmetic subgroups of semisimple Lie groups are defined using algebraic groups. This way the classification of semisimple algebraic groups [Tit66] implies a classification of the possible types of arithmetic subgroups. It follows from the classification that for hyperbolic spaces of even dimension all arithmetically defined subgroups are arithmetic subgroups of the simplest type. For odd $n$ there is another family of arithmetic subgroups given as the groups of units of appropriate Hermitian forms over quaternion algebras. Moreover, if $n=7$, there is also the third type of arithmetic subgroups of $H$ which are associated to the Cayley algebra. The following lemma of Vinberg shows that for our purpose it will be always sufficient to consider only arithmetic subgroups of the simplest type:

Lemma 2.1 ( Vin67, Lemma 7]). Any arithmetic lattice $\Gamma \subset H$ generated by reflections is an arithmetic lattice of the simplest type.

A discrete subgroup of a Lie group $H$ is called maximal if it is not properly contained in any other discrete subgroup of $H$. It is well known that in semisimple Lie groups any lattice is contained in a maximal lattice. Let $\Gamma$ be an arithmetic subgroup which is commensurable with $\mathrm{G}\left(\mathfrak{o}_{k}\right)=\mathrm{O}\left(f, \mathfrak{o}_{k}\right)$ for some quadratic form $f$ defined over $k$. There exists an $\mathfrak{o}_{k}$-lattice $L$ in $k^{n+1}$ such that $\Gamma \cap \mathrm{G}(k) \subset \mathrm{G}^{L}=$ $\{g \in \mathrm{G}(k) \mid g(L)=L\}$ (cf. [Vin71]). If $\mathfrak{o}_{k}$ is a principal ideal domain in an appropriate basis, the transformations from $\mathrm{G}^{L}$ can be written down by matrices with elements in $\mathfrak{o}_{k}$. By Theorem 5 from Vin71, if $\Lambda<\Gamma$ is an arithmetic subgroup generated by reflections, then $\Lambda$ is definable over $\mathfrak{o}_{k}$. We refer to [Vin71] for more 
information about the rings of definition of arithmetic subgroups. Note that the term "field of definition" is used by Vinberg in a more restricted sense.

A certain subclass of arithmetic subgroups will play a special role in our study. An arithmetic subgroup $\Gamma<\mathrm{O}_{0}(f)$ is called a congruence subgroup if there exists a non-zero ideal $\mathfrak{a} \subset \mathfrak{o}_{k}$ such that $\Gamma \supset \mathrm{O}_{0}(f, \mathfrak{a})$, where $\mathrm{O}_{0}(f, \mathfrak{a})=\left\{g \in \mathrm{O}_{0}\left(f, \mathfrak{o}_{k}\right) \mid g \equiv\right.$ Id $(\bmod \mathfrak{a})\}$ is the principal congruence subgroup of $\mathrm{O}_{0}\left(f, \mathfrak{o}_{k}\right)$ of level $\mathfrak{a}$. Congruence subgroups possess a remarkable set of special geometric and algebraic properties. In particular, the first non-zero eigenvalue of the Laplacian on $\mathbb{H}^{n} / \Gamma$ is bounded away from zero by a constant which depends only on $n$ but not on $\Gamma$. This spectral gap property will play a key role in the methods that we are going to discuss in Sections 4 and 5 .

We have the following fact connecting maximal and congruence arithmetic subgroups:

\section{Lemma 2.2. Maximal arithmetic subgroups are congruence.}

For the arithmetic subgroups defined by quadratic forms, the proof of the lemma can be found in [ABSW08, Lemma 4.7], where it is based on a material from [PR94.

An interested reader can find much more information about arithmetic groups and their properties in the books [PR94] and Wit15].

Let us now come back to the reflection groups - the objects of our primary interest. A reflection group $\Gamma$ is called a maximal reflection group if there is no other discrete subgroup $\Gamma^{\prime}<\operatorname{Isom}\left(\mathbb{H}^{n}\right)$ such that $\Gamma<\Gamma^{\prime}$ and $\Gamma^{\prime}$ is generated by reflections. Maximal reflection groups are not necessarily maximal lattices but there is a relation between the two, and it is captured by the following lemma due to Vinberg:

Lemma 2.3. A maximal reflection group $\Gamma$ is a normal subgroup of a maximal lattice $\Gamma_{0}$. Moreover, there is a finite subgroup $\Theta<\Gamma_{0}$ such that $\Theta \rightarrow \Gamma_{0} / \Gamma$ is an isomorphism, and $\Theta$ is the group of symmetries of the Coxeter polyhedron of $\Gamma$.

This lemma was proved in Vin67, the argument is also reproduced in ABSW08.

Given an admissible quadratic form $f$ as above, we would like to know when the arithmetic subgroup $\Gamma=\mathrm{O}_{0}\left(f, \mathfrak{o}_{k}\right)$ is, up to finite index, generated by hyperbolic reflections. If this is the case, the form $f$ and the group $\Gamma$ are called reflective. The main practical tool for deciding reflectivity is Vinberg's algorithm [Vin72, which we are going to review now.

In the vector model of $\mathbb{H}^{n}$, a hyperplane is given by the set of rays in $\mathfrak{C}$ which are orthogonal to a vector $e$ of positive square in $\mathbb{E}^{n, 1}$. A hyperplane $\Pi_{e}$ defines two half-spaces, $\Pi_{e}^{+}$and $\Pi_{e}^{-}$, where ${ }^{\prime} \pm^{\prime}$ is the sign of $(e, x)$ for $x$ in the corresponding half-space, and a reflection

$$
R_{e}: x \rightarrow x-2 \frac{(e, x)}{(e, e)} e
$$

where the inner product $(u, v)=\frac{1}{2}(f(u+v)-f(u)-f(v))$ is induced by $f$.

We shall assume that $\mathfrak{o}_{k}$ is a principal ideal domain (PID). The vector $e$ corresponding to the reflection $R_{e}$ is defined up to scaling, so if $e$ has $k$-rational coordinates, we can normalize it so that the coordinates are coprime integers in $\mathfrak{o}_{k}$. With this normalization we can assign to $R_{e}$ a parameter $s=(e, e) \in \mathfrak{o}_{k}$, and call $R_{e}$ an $s$-reflection. The reflection $R_{e}$ belongs to the group $\mathrm{O}_{0}\left(f, \mathfrak{o}_{k}\right)$ if $\frac{2}{s}\left(e, v_{i}\right) \in \mathfrak{o}_{k}$, for the standard basis vectors $v_{i}, i=0, \ldots, n$. Following [Vin72], we call this the crystallographic condition. 
We begin by considering the stabilizer subgroup of a vector $u_{0}$ with integral coordinates which corresponds to a point $x_{0} \in \overline{\mathbb{H}^{n}}$. (When dealing with noncocompact lattices it may be convenient to choose an ideal point $x_{0} \in \partial \mathbb{H}^{n}$ as a starting node.) Consider the (finite) group generated by all reflections in $\Gamma$ whose mirrors pass through $x_{0}$. Let

$$
\mathrm{P}_{0}=\bigcap_{i=1}^{m} \Pi_{e_{i}}^{-}
$$

be a fundamental chamber of this group. All the half-spaces $\Pi_{e_{i}}^{-}$are essential (i.e., not containing the intersection of the other half-spaces). The corresponding vectors $e_{i}$ satisfy $\left(e_{i}, e_{i}\right)>0,\left(e_{i}, u_{0}\right)=0$ for all $i$, and the reflections $R_{e_{i}}$ generate the stabilizer of $x_{0}$ in $\mathrm{O}_{0}\left(f ; \mathfrak{o}_{k}\right)$. There is a unique fundamental polyhedron $\mathrm{P}$ of the reflection subgroup which sits inside $\mathrm{P}_{0}$ and contains $x_{0}$. The point $x_{0}$ is not necessarily a vertex of $\mathrm{P}$ but it is usually convenient to choose $u_{0}$ in such a way that $x_{0}$ is a vertex.

The algorithm continues by picking up further $\Pi_{e_{i}}$ so that

$$
\mathrm{P} \subseteq \bigcap_{i} \Pi_{e_{i}}^{-}
$$

This is done by choosing $e_{i}$ satisfying the crystallographic condition such that $\left(e_{i}, e_{i}\right)>0,\left(e_{i}, u_{0}\right)<0,\left(e_{i}, e_{j}\right) \leq 0$ for all $j<i$, and the distance between $x_{0}$ and $\Pi_{e_{i}}$ is the smallest possible, i.e., minimizing the value

$$
\sinh ^{2}\left(\operatorname{dist}\left(x_{0}, \Pi_{e_{i}}\right)\right)=-\frac{\left(e_{i}, u_{0}\right)^{2}}{\left(e_{i}, e_{i}\right)\left(u_{0}, u_{0}\right)} .
$$

The latter condition implies that all the hyperplanes $\Pi_{e_{i}}$ are essential. Note that if $k \neq \mathbb{Q}$, its integers do not form a discrete subset of $\mathbb{R}$. Bugaenko showed that regardless of this, the arithmeticity assumption implies that the set of distances considered above is discrete and hence we can always choose the smallest one (see Bug84, Bug90, Bug92, or Mar15b).

The algorithm terminates if it generates a configuration $\mathrm{P}=\bigcap_{i} \Pi_{e_{i}}^{-}$that has finite volume, in which case the form $f$ is reflective. The finite volume condition can be effectively checked from the Coxeter diagram of $\mathrm{P}$ - it is equivalent to each edge of the polyhedron having two vertices, either one or both of which may be at the ideal boundary of the hyperbolic space.

Let us recall that the fundamental polyhedra of the reflection groups are usually described using Coxeter diagrams. These are the graphs with vertices corresponding to the vectors $e_{i}$ (equivalently, the faces of $\mathrm{P}$ ). Two different vertices $e_{i}, e_{j}$ are connected by a thin edge of integer weight $m_{i j} \geq 3$ or by $m_{i j}-2$ edges if the corresponding faces intersect with the dihedral angle $\frac{\pi}{m_{i j}}$, by a thick edge if they intersect at infinity (dihedral angle zero), and by a dashed edge if they are divergent. In particular, two vertices are not joined by an edge if and only if the corresponding faces of $\mathrm{P}$ are orthogonal. Note that there are some differences between the Coxeter diagrams and the Dynkin diagrams which are used in Lie theory. In particular, the triple edge in our labeling convention means the angle $\frac{\pi}{5}$, while on the Dynkin diagram of a Weyl chamber it corresponds to the angle $\frac{\pi}{6}$. 
In order to produce the Coxeter diagram of the polyhedron $\mathrm{P}$, we can compute the dihedral angles between the intersecting hyperplanes from the standard formula

$$
\cos \left(\frac{\pi}{m_{i j}}\right)=\frac{-\left(e_{i}, e_{j}\right)}{\sqrt{\left(e_{i}, e_{i}\right)\left(e_{j}, e_{j}\right)}} .
$$

Example 2.4. Let us consider the quadratic form

$$
f=2\left(x_{1}^{2}+x_{2}^{2}+x_{3}^{2}+x_{4}^{2}-x_{1} x_{2}-\frac{1}{2}(1+\sqrt{5}) x_{2} x_{3}-x_{3} x_{4}\right) .
$$

It is an admissible even quadratic form of the discriminant $3-2 \sqrt{5}$ defined over the field $k=\mathbb{Q}(\sqrt{5})$.

We can start running the algorithm with $u_{0}=\left(\frac{3}{2}, 3,2 \phi, \phi\right)$, where $\phi=\frac{1+\sqrt{5}}{2}$ is the fundamental unit of $k$, so that the stabilizer subgroup of the corresponding point $x_{0} \in \mathbb{H}^{3}$ is generated by reflections corresponding to the vectors

$$
e_{1}=(1,0,0,0), e_{2}=(0,0,1,0), \text { and } e_{3}=(0,0,0,1) .
$$

The algorithm finds the fourth vector $e_{4}=(-1,-1,-\phi,-\phi)$ and terminates.

The Coxeter diagram of the resulting configuration is

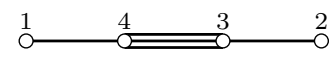

The polyhedron $\mathrm{P}$ is a bounded simplex in $\mathbb{H}^{3}$, and the group $\Gamma$ is generated by reflections in its sides is a well known arithmetic lattice. A difficult theorem of Gehring, Martin, and Marshall GM09, MM12 shows that the order 2 extension of $\Gamma$ is the minimal covolume lattice in $\operatorname{Isom}\left(\mathbb{H}^{3}\right)$; hence, we can think of it as the three-dimensional analogue of the $(2,3,7)$-Hurwitz group.

Note that the form $f$ is not diagonalizable over $\mathfrak{o}_{k}$, and it can be verified that the group $\Gamma$ cannot be obtained as a reflection subgroup of the group of units of some diagonal quadratic form.

We refer to Vin72,VK78, Bug84, Bug90,Sha90, Bug92, SW92, Nik00, All12,BM13, Mcl13 Mar15a, Mar15b for many other examples of the Coxeter polyhedra and their diagrams produced by the algorithm. In most of these papers the form $f$ is defined over $\mathbb{Q}$ which implies (for $n \geq 4$ ) that the resulting Coxeter polyhedra have cusps. The exceptions are the Bugaenko papers where the algorithm was first applied to the forms with coefficients in the real quadratic fields leading to examples of cocompact arithmetic hyperbolic reflection groups in dimensions $n \leq 8$. We shall come back to the discussion of the known examples in Section 7

Let us point out that Vinberg's algorithm has an unfortunate property that it never halts if the form is not reflective. Fortunately, in practice this problem can be often bypassed: if a computer implementation of the algorithm produces, say, more than $10^{3}$ generators for the reflection subgroup, we can expect that it is not a lattice. The latter condition can be rigorously checked by the group theoretic methods in each particular case, for example, by detecting an infinite order symmetry of the reflection polyhedron, it then implies that the group was not reflective. Many concrete examples and some general methods for this verification can be found in the papers cited above. 


\section{THE WORK OF NIKULIN}

In a series of papers beginning with the 1980 article Nik80, Nikulin established finiteness of the number of commensurability classes of arithmetic hyperbolic reflection groups and obtained upper bounds for degrees of their fields of definition. In this section, we shall briefly review Nikulin's method.

Let $\mathrm{P}$ be a convex polyhedron in $\mathbb{H}^{n}$. It is an intersection of finitely many halfspaces $\Pi_{e_{i}}^{-}$, where the vectors $e_{i}$ orthogonal to the faces of $\mathrm{P}$ can be chosen to have square 2 and directed outward. The matrix

$$
A(P)=\left(a_{i j}\right)=\left(e_{i}, e_{j}\right)
$$

is called the Gram matrix of P. It uniquely determines $\mathrm{P}$ up to motions of the ambient space $\mathbb{H}^{n}$.

The polyhedron $\mathrm{P}$ is a fundamental polyhedron of a discrete reflection group in $\mathbb{H}^{n}$ if and only if $a_{i j} \leq 0$ and $a_{i j}=-2 \cos \left(\frac{\pi}{m_{i j}}\right)$, where $m_{i j} \geq 2$ is an integer whenever $a_{i j}>-2$ for all $i \neq j$. Symmetric real matrices $A$ satisfying these conditions and having all their diagonal elements equal to 2 are called the fundamental matrices.

Note. In his papers, Nikulin uses the opposite sign convention which is more common in algebraic geometry. Here we keep the notation that was introduced in the previous section, which is also consistent with the one used by Vinberg.

Given a real $t>0$, we say that the fundamental Gram matrix $A=\left(a_{i j}\right)$ and the corresponding polyhedron $\mathrm{P}$ have minimality $t$ if $\left|a_{i j}\right|<t$ for all $a_{i j}$. We can analogously define the minimality of a face $\mathrm{F}$ of $\mathrm{P}$ by considering the Gram matrix $A(\mathrm{~F})$ formed by the inner products of the vectors associated to the faces of $\mathrm{P}$ that have non-trivial intersections with $\mathrm{F}$ in $\overline{\mathbb{H}^{n}}$. The notion of minimality is central for Nikulin's method.

Suppose that $\mathrm{P}$ is a fundamental polyhedron of an arithmetic reflection group $\Gamma=\Gamma(\mathrm{P})$ in $\mathbb{H}^{n}$. Vinberg Vin67] proved that for a finite volume polyhedron $\mathrm{P}$ it is equivalent to the conditions that all the cyclic products

$$
b_{i_{1} \cdots i_{m}}=a_{i_{1} i_{2}} a_{i_{2} i_{3}} \cdots a_{i_{m-1} i_{m}} a_{i_{m} i_{1}}
$$

are algebraic integers, the field $K=\mathbb{Q}\left(\left\{a_{i j}\right\}\right)$ is totally real, and the matrices $A^{\sigma}=\left(a_{i j}^{\sigma}\right)$ are non-negative definite for every embedding $\sigma: K \rightarrow \mathbb{R}$ not equal to identity on the field of definition $k=\mathbb{Q}\left(\left\{b_{i_{1} \cdots i_{m}}\right\}\right)$, which is generated by the cyclic products.

A fundamental matrix $A(\mathrm{P})$ and the corresponding reflection group $\Gamma(\mathrm{P})$ is called $V$-arithmetic if it satisfies the conditions of Vinberg's arithmeticity criterion except that $\mathrm{P}$ is not required to have finite volume. The property of $V$-arithmeticity is much easier to check than arithmeticity and, moreover, it is inherited by subpolyhedra: if $\mathrm{P}^{\prime}$ is an intersection of a subset of the half-spaces $\Pi_{e_{i}}^{-}$defining the polyhedron $\mathrm{P}$ and its Gram matrix $A\left(\mathrm{P}^{\prime}\right)$ is indefinite, then $\mathrm{P}^{\prime}$ is also $V$-arithmetic with the same field of definition (also called ground field) as $\mathrm{P}$.

The hereditary property of $V$-arithmeticity allows us to reduce some questions to the analysis of simple configurations. The basic case is called an edge polyhedron (chamber). It refers to a fundamental matrix $A(\mathrm{P})$ such that all the corresponding hyperplanes $\Pi_{e}$ contain at least one of the two distinct vertices $v_{1}, v_{2}$ of a onedimensional edge $v_{1} v_{2}$ of $\mathrm{P}$. If the vertices $v_{1}, v_{2}$ are finite, the edge chamber 
is called finite. In this part we are mainly interested in bounding the degree of the fields of definition of arithmetic reflection groups, thus we can restrict to the finite case. With the necessary modifications, Nikulin's method also applies to the unbounded Coxeter polyhedra. Any finite edge chamber has precisely $n+1$ sides corresponding to the vectors $e_{1}, e_{2}$ and $n-1$ vectors $\left\{e_{j}\right\}_{j \in J}$ whose hyperplanes contain the full edge $v_{1} v_{2}$. The Gram matrix $A(\mathrm{P})$ is hyperbolic but its submatrices corresponding to $e_{1} \cup\left\{e_{j}\right\}_{j \in J}$ and $e_{2} \cup\left\{e_{j}\right\}_{j \in J}$ are positive definite. The only element of $A(\mathrm{P})$ that can have absolute value bigger than 2 is $u=\left(e_{1}, e_{2}\right)$. It follows that an edge polyhedron has minimality $t>2$ if and only if $|u|=\left|\left(e_{1}, e_{2}\right)\right|<t$. The Coxeter diagram $G$ of an edge chamber $\mathrm{P}$ has exactly one connected component $G\left(\mathrm{P}^{\text {hyp }}\right)$ corresponding to a hyperbolic submatrix of $A(\mathrm{P})$ and containing $e_{1}$ and $e_{2}$, and possibly several positive definite connected components. The Gram matrix $A\left(\mathrm{P}^{\text {hyp }}\right)$ corresponds to an edge chamber of dimension $\# G\left(\mathrm{P}^{\text {hyp }}\right)-1$. If $\mathrm{P}$ is $V$ arithmetic, then $\mathrm{P}$ and the hyperbolic connected component $\mathrm{P}$ hyp are defined over the same field $k$.

We now can state a principal technical theorem of Nikulin:

Theorem 3.1 ([Nik81a, Theorem 2.3.1]). Given any $t>0$, there is an effective constant $N(t)$ such that for every $V$-arithmetic edge chamber of minimality $t$ with the ground field $k$ of degree more than $N(t)$ over $\mathbb{Q}$, the number of vertices in the hyperbolic connected component of the Coxeter graph is less than 4.

The proof of Theorem 3.1 in Nik81a] uses a variant of Fekete's theorem (1923) on the existence of non-zero integer polynomials of bounded degree with small deviation from zero on appropriate intervals (see also [Nik11, Section 6] for a review of the proof and some corrections).

The minimality $t=14$ is especially important for Nikulin's method. This is because a fundamental polyhedron of an arithmetic hyperbolic reflection group (not assumed to be cocompact here) always has a face with minimality 14-a result that was proved in Nikulin's early papers (see [Nik80, Lemma 3.2.1] and Nik81a, proof of Theorem 4.1.1]). This fact allows one to reduce various finiteness and classification problems to the estimation of the value of the transition constant $N(14)$. This way in [Nik07], Nikulin showed that his previous work together with the finiteness results for dimensions $n=2$ and 3 obtained by the spectral method (to be discussed in the next section) can be applied to prove a general finiteness theorem for the number of commensurability classes of arithmetic hyperbolic reflection groups. He also proved that the degrees of the fields of definition of arithmetic hyperbolic reflection groups are bounded above by the maximum of $N(14)$ and the maximal possible degree in dimensions 2 and 3. In the subsequent papers Nikulin gave explicit bounds for the constant $N(14)$, for example, in [Nik09] (see also Remark 5.1 in [Nik11] for a correction) he showed that $N(14) \leq 120$. The best result of this kind is obtained in [Nik11], where it is shown that $N(14) \leq 25$. It is worth mentioning that most of these results make use of Theorem 3.1 and its proof. The main difference between the latest improvement and the previous papers is that there, instead of relying only on estimates for the Fekete's existence theorem, Nikulin constructed certain explicit polynomials with the required properties.

The result of [Nik11], together with [Mac11] and [BL14], which we shall discuss later on in Section [5 implies: 
Theorem 3.2 (cf. [BL14, Corollary 5.1]). The degree of the fields of definition of arithmetic hyperbolic reflection groups in all dimensions is at most 25.

This is currently the best known general bound.

\section{Spectral method And finiteness theorems}

Given a lattice $\Gamma \leq \operatorname{Isom}\left(\mathbb{H}^{n}\right)$, we have an associated quotient Riemannian orbifold $\mathcal{O}=\mathbb{H}^{n} / \Gamma$. If $\Gamma$ is generated by reflections, we call $\mathcal{O}$ a reflection orbifold. The main idea of the method discussed in this section is that the global geometric properties of the reflection orbifolds can provide important information about the hyperbolic reflection groups.

The spectral method that we are going to review is based on some properties of the spectrum of the Laplacian on orbifolds. It was first applied for proving finiteness results for arithmetic hyperbolic reflection groups by Long, Maclachlan, and Reid LMR06 and by Agol Ago06 in dimensions $n=2$ and 3, respectively. The first paper makes use of the Zograf's spectral proof of Rademacher's conjecture for congruence subgroups of the modular group Zog91. In his work [Ago06, Agol found that one can employ the Li-Yau inequality for conformal volume instead of Zograf's spectral inequality for surfaces. This allows us to extend the domain of applicability of the method to a much wider class of spaces. Agol completed the proof of the finiteness theorem for $n=3$; a later joint work with Agol, Belolipetsky, Storm, and Whyte showed how to extend the argument to an arbitrary dimension ABSW08. We shall now review this approach.

Conformal volume was introduced by Li and Yau in [LY82, partially motivated by generalizing results on surfaces due to Yang and Yau YY80, Hersch Her70, and Szegö [Sze54]. In ABSW08, we generalized this notion to orbifolds. Let $(\mathcal{O}, g)$ be a complete Riemannian orbifold, possibly with boundary, and let $|\mathcal{O}|$ denote the underlying topological space. Denote the volume form by $d v_{g}$, and the volume by $\operatorname{Vol}(\mathcal{O}, g)$. Let $\operatorname{Mob}\left(\mathbb{S}^{n}\right)$ denote the group of conformal transformations of $\mathbb{S}^{n}$. It is well known that $\operatorname{Mob}\left(\mathbb{S}^{n}\right)=\operatorname{Isom}\left(\mathbb{H}^{n+1}\right)$. The topological space $|\mathcal{O}|$ has a dense open subset which is a Riemannian manifold. We call a map $\varphi:\left|\mathcal{O}_{1}\right| \rightarrow\left|\mathcal{O}_{2}\right|$ a $P C$ $m a p$ if it is a continuous map which is piecewise a conformal immersion. Clearly, if $\varphi:|\mathcal{O}| \rightarrow \mathbb{S}^{n}$ is $\mathrm{PC}$, and $\mu \in \operatorname{Mob}\left(\mathbb{S}^{n}\right)$, then $\mu \circ \varphi$ is also a $\mathrm{PC}$ map.

Let $\left(\mathbb{S}^{m}\right.$, can $)$ be the $m$-dimensional sphere with the canonical round metric. For a piecewise smooth map $\varphi:|\mathcal{O}| \rightarrow\left(\mathbb{S}^{m}\right.$, can $)$, define

$$
V_{P C}(m, \varphi)=\sup _{\mu \in \operatorname{Mob}\left(\mathbb{S}^{m}\right)} \operatorname{Vol}\left(\mathcal{O},(\mu \circ \varphi)^{*}(\text { can })\right) .
$$

If there exists a PC map $\varphi:|\mathcal{O}| \rightarrow \mathbb{S}^{m}$, then we also define

$$
V_{P C}(m, \mathcal{O})=\inf _{\varphi:|\mathcal{O}| \rightarrow \mathbb{S}^{m} P C} V_{P C}(m, \varphi) .
$$

We call $V_{P C}(m, \mathcal{O})$ the $m$-dimensional piecewise conformal volume of $\mathcal{O}$.

Using the Nash embedding theorem, it can be shown that the $m$-dimensional conformal volume is always well defined for a sufficiently large $m$. It is clear that $V_{P C}(m, \mathcal{O}) \geq V_{P C}(m+1, \mathcal{O})$, hence we can define the (piecewise) conformal volume

$$
\operatorname{Vol}_{c}(\mathcal{O})=\lim _{n \rightarrow \infty} V_{P C}(n, \mathcal{O}) .
$$

We refer to ABSW08 for further discussion and basic properties of the conformal volume. One of the immediate corollaries of the definitions allows us to compute 
the conformal volume of the reflective orbifolds (cf. Facts 3, 4 in ABSW08, Section $2])$ :

Let $\Gamma_{r}<\operatorname{Isom}\left(\mathbb{H}^{n}\right)$ be a lattice generated by reflections in hyperplanes in $\mathbb{H}^{n}$, and let $\mathcal{O}_{r}=\mathbb{H}^{n} / \Gamma_{r}$. Then we have $\operatorname{Vol}_{c}\left(\mathcal{O}_{r}\right)=$ $\operatorname{Vol}\left(\mathbb{S}^{n}\right.$, can $)$.

Now the Li-Yau inequality generalized to $n$-dimensional orbifolds says ABSW08:

$$
\lambda_{1}(\mathcal{O}) \cdot \operatorname{Vol}(\mathcal{O})^{\frac{2}{n}} \leq n \cdot \operatorname{Vol}_{c}(\mathcal{O})^{\frac{2}{n}} .
$$

Here $\lambda_{1}(\mathcal{O})$ denotes the first non-zero eigenvalue of the Laplacian on $\mathcal{O}$ (called the spectral gap), Vol is the hyperbolic volume, and $\mathrm{Vol}_{c}$ is the conformal volume defined above.

Equation (11) shows that information about the spectral gap and hyperbolic volume of a reflection orbifold can be played against the upper bound given by the value of its conformal volume. The information that we need here can be deduced from arithmeticity. Indeed, if $\Gamma$ is a congruence subgroup of $\operatorname{Isom}\left(\mathbb{H}^{n}\right)$ (cf. Section 2 for the terminology), then the well known conjectures of Ramanujan and Selberg imply that

$$
\lambda_{1}\left(\mathbb{H}^{n} / \Gamma\right) \geq \lambda_{1}\left(\mathbb{H}^{n}\right) .
$$

These conjectures are still open, but less precise low bounds are known, and for our purpose they can serve almost as well as the conjectures. We have

$$
\lambda_{1}\left(\mathbb{H}^{n} / \Gamma\right) \geq \delta(n),
$$

where $\delta(2)=\frac{3}{16}$ by Vigneras Vig83 and if $n \geq 3, \delta(n)=\frac{2 n-3}{4}$ by Burger and Sarnak BS91. Moreover, if $\Gamma$ is defined by a quadratic form (which is always the case for the arithmetic hyperbolic reflection groups by Lemma 2.1), then more recent work of Luo, Rudnick, and Sarnak implies that we can take $\delta(2)=0.21$ and $\delta(n)=\frac{15 n-24}{25}$ for $n \geq 3$ [LRS99]. The proofs of these bounds are based on deep results about automorphic representations.

Now let us assume that $\Gamma$ is at the same time a congruence subgroup and a reflection group, and let $\mathcal{O}=\mathbb{H}^{n} / \Gamma$. Following the argument in Bel11, we can then quickly prove the two principal finiteness theorems. We have:

$$
\begin{gathered}
\delta(n) \cdot \operatorname{Vol}(\mathcal{O})^{\frac{2}{n}} \leq n \cdot \operatorname{Vol}\left(\mathbb{S}^{n}\right)^{\frac{2}{n}} ; \\
\operatorname{Vol}(\mathcal{O}) \leq\left(\frac{n}{\delta(n)}\right)^{\frac{n}{2}} \operatorname{Vol}\left(\mathbb{S}^{n}\right) .
\end{gathered}
$$

By the theorems of Wang Wan72 for $n \geq 4$ and Borel Bor81 for $n=2,3$, there are only finitely many (up to conjugacy) arithmetic subgroups of $\operatorname{Isom}\left(\mathbb{H}^{n}\right)$ of bounded covolume. As the right-hand side of (2) depends only on the dimension, we immediately obtain our first finiteness theorem:

Theorem 4.1. For every $n \geq 2$ there are only finitely many conjugacy classes of congruence reflection subgroups of $\operatorname{Isom}\left(\mathbb{H}^{n}\right)$.

Let $n \geq 3$. We have $\delta(n) \geq \frac{15 n-24}{25}$;

$$
\frac{\operatorname{Vol}(\mathcal{O})}{\operatorname{Vol}\left(\mathbb{S}^{n}\right)} \leq\left(\frac{25 n}{15 n-24}\right)^{\frac{n}{2}} \leq\left(\frac{25}{7}\right)^{\frac{n}{2}}
$$

At this place we need to recall some recent results about volumes of arithmetic hyperbolic $n$-orbifolds. By Bel04, Bel07, and $\mathrm{BE12}, \operatorname{Vol}\left(\mathbb{H}^{n} / \Gamma\right)$ is bounded 
below by a function which grows super-exponentially with $n$. These results are discussed in some detail the survey article Bel14; the required corollary in a more precise form can be found as Corollary 3.3 there. As $\operatorname{Vol}\left(\mathbb{S}^{n}\right) \rightarrow 0$ when $n \rightarrow \infty$, the super-exponential lower bound holds true also for the quotient $\operatorname{Vol}\left(\mathbb{H}^{n} / \Gamma\right) / \operatorname{Vol}\left(\mathbb{S}^{n}\right)$. Hence the left-hand side of (3) grows super-exponentially with $n$ while the righthand side is only exponential. This gives our second finiteness theorem:

Theorem 4.2. If $n$ is sufficiently large, then $\operatorname{Isom}\left(\mathbb{H}^{n}\right)$ does not contain any congruence reflection subgroups.

The main problem with this argument is that we cannot expect that all maximal arithmetic reflection groups are congruence - the counterexamples are known in dimensions 2 and 3 by the work of Lakeland [Lak12a, Lak12b], and it is not clear what happens in higher dimensions. The goal of ABSW08 was to show how finer arithmetic techniques can be applied in order to partially bypass this difficulty and prove an analogue of Theorem 4.1 for all maximal arithmetic reflection groups. We cannot yet prove an analogue of Theorem 4.2 using the spectral method, but we know that it is true thanks to the previous work of Vinberg (cf. Theorem 1.1 in the Introduction). Thus we have:

Theorem 4.3. There are only finitely many conjugacy classes of arithmetic maximal hyperbolic reflection groups.

This result was proved independently in ABSW08 and [Nik07. It implies that in principle it is possible to give a complete classification of the arithmetic hyperbolic reflection groups.

Let us note that neither arithmeticity nor maximality assumptions in Theorem 4.3 can be dropped. Examples of infinite families of arithmetic reflection groups up to dimension 19 have been given by Allcock [All06. These examples are obtained using the idea of doubling: if a fundamental polyhedron $\mathrm{P}$ of a reflection group $\Gamma$ has a face $\mathrm{F}$ whose all dihedral angles with the other faces are equal to $\frac{\pi}{2}$, then we can double $\mathrm{P}$ along $\mathrm{F}$ to obtain a new polyhedron such that the reflections in its faces generate an index 2 subgroup of $\Gamma$ (see Section 7 for a precise description of Allcock's redoubling procedure and a related discussion). It is clear that the groups which are obtained by this procedure and its variations are non-maximal reflection groups. The necessity of the arithmeticity assumption is also well known. For example, consider the groups generated by reflections of the hyperbolic plane in the sides of a hyperbolic triangle with angles $\frac{\pi}{2}, \frac{\pi}{3}, \frac{\pi}{m}(m \geq 7)$. These groups are known to be maximal discrete subgroups of $\operatorname{Isom}\left(\mathbb{H}^{2}\right)$, but all except finitely many of them are non-arithmetic. A similar construction is available for hyperbolic three-space with triangular prisms replacing triangles (see [MR03, Section 10.4.3]).

Finally, let us mention that the spectral method we described in this section has other interesting variations and applications. We refer to Peter Sarnak's lecture notes Sar14] for an enlightening discussion of some related topics.

\section{EfFective REsults obtained By the SPECtral Method}

The proofs from the previous section can be made effective, meaning that we can essentially enumerate all the possible candidates for the reflection groups in Theorems 4.1 and 4.2. In order to do so, we need to look at the quantitative side of the finiteness theorems of Borel and Wang. The key ingredient for quantitative 
analysis is provided by the results on minimal volume arithmetic hyperbolic $n$ orbifolds Bel04,Bel07,BE12, (see also Bel14]) and the methods from these papers. In particular, all these results were obtained using the volume formula of G. Prasad Pra89, which will also play a prominent role in our discussion.

More precisely, the quantitative analogue of Theorem 4.2 is:

Proposition 5.1 (cf. [Bel11, Prop. 4.1]). There are no cocompact congruence reflection subgroups in $\operatorname{Isom}\left(\mathbb{H}^{n}\right)$ for $n \geq 13$, and no congruence reflection subgroups in $\operatorname{Isom}\left(\mathbb{H}^{n}\right)$ for $n \geq 28$.

It is proved by substituting in (3) the precise lower bounds for the minimal volume. With some case-by-case considerations it should not be hard to bring the second bound down to 22, so that it would agree with the Esselmann's result Ess96. bounding the dimension of non-cocompact arithmetic reflection groups.

A finer analysis based on volume computations leads to the quantitative version of Theorem 4.2 .

Proposition 5.2 (cf. Bel11, Prop. 4.2]). The degrees of the fields of definition of cocompact congruence reflection subgroups of $\mathrm{PO}(n, 1)$ are bounded by 6 and their discriminants satisfy the conditions in Table 1.

TABLE 1. The bounds for $\mathcal{D}_{k}$ depending on the dimension $n$ and the degree of the field $d=[k: \mathbb{Q}]$.

\begin{tabular}{r|ccccc} 
& $d=2$ & 3 & 4 & 5 & 6 \\
\hline$n=4$ & $\leq 262$ & $\leq 2244$ & $\leq 19210$ & $\leq 164442$ & $\leq 1407650$ \\
5 & $\leq 214$ & $\leq 1928$ & $\leq 17302$ & $\leq 155272$ & $\leq 1393406$ \\
6 & $\leq 28$ & 49,81 & & & \\
7 & $\leq 39$ & $\leq 205$ & $\leq 1062$ & & \\
8,9 & $\leq 13$ & & & & \\
10,11 & 5,8 & & & & \\
12 & 5 & & & &
\end{tabular}

Similar methods can be employed to look at the other invariants (the HasseWitt symbol and the determinant of the quadratic form $f$ defining $\Gamma$ ) with an objective to give a list containing all the congruence-reflective quadratic forms. However, this has not been done yet. In small dimensions the list will be very large, but for higher $n$ its size will reduce quickly - compare with the possible fields of definition in Proposition 5.2. Producing the list of the quadratic forms is a feasible task which would provide important data for the potential classification of arithmetic hyperbolic reflection groups. We shall come back to this discussion later in Section 10.

The main issue about the results of the propositions is that they depend on an extra assumption - the maximal reflection groups have to be congruence. We do not know yet how much, if any, information we loose by imposing this condition in dimensions $n \geq 4$ but for $n=2$ and 3, we do have Lakeland's examples showing that not all arithmetic maximal reflection groups have this property Lak12a, Lak12b]. Fortunately, there is a way to prove effective results in small dimensions without restricting to the congruence subgroups. Let us now review this method. 
The idea goes back to [LMR06] and Ago06. From the point of view based on conformal volume, we can explain it as Agol's trick:

Let $\Gamma_{0}<\operatorname{Isom}\left(\mathbb{H}^{n}\right)$ be a maximal arithmetic subgroup containing a maximal reflection group $\Gamma$. Since a conjugation of a reflection in $\operatorname{Isom}\left(\mathbb{H}^{n}\right)$ is again a reflection, the subgroup $\Gamma$ is normal in $\Gamma_{0}$. By Vinberg's lemma (cf. Lemma 2.3) we have that the quotient group $\Gamma_{0} / \Gamma$ is isomorphic to a finite subgroup $\Theta<\Gamma_{0}$ which is the group of symmetries of the Coxeter polyhedron $\mathrm{P}$ of $\Gamma$. Consider $\mathbb{H}^{n} \subset \mathbb{S}^{n}$ embedded conformally as the upper half-space of $\mathbb{S}^{n}$, so that $\operatorname{Isom}\left(\mathbb{H}^{n}\right)$ acts conformally on $\mathbb{S}^{n}$. Normalize so that $\Theta$ acts isometrically on $\mathbb{S}^{n}$. Clearly, $V_{P C}(m, \mathcal{O})=V_{P C}(m, \mathrm{P} / \Theta)$, where $\mathcal{O}=\mathbb{H}^{n} / \Gamma_{0}$, and so $|\mathcal{O}|=|\mathrm{P} / \Theta|$. The orbifold embedding $\mathrm{P} / \Theta \subset \mathbb{S}^{n} / \Theta$ is a conformal embedding; hence, by one of the basic properties of the conformal volume, we have $V_{P C}(n, \mathrm{P} / \Theta) \leq V_{P C}\left(n, \mathbb{S}^{n} / \Theta\right)$. The key observation is that we can give a good upper bound for $V_{P C}\left(n, \mathbb{S}^{n} / \Theta\right)$ if we manage to embed $\Theta$ in a finite reflection group $\Theta^{\prime}$. Indeed, in this case we have

$$
V_{P C}\left(n, \mathbb{S}^{n} / \Theta\right) \leq\left[\Theta^{\prime}: \Theta\right] \cdot V_{P C}\left(n, \mathbb{S}^{n} / \Theta^{\prime}\right) \quad \text { and } \quad V_{P C}\left(n, \mathbb{S}^{n} / \Theta^{\prime}\right)=\operatorname{Vol}\left(\mathbb{S}^{n}\right),
$$

which leads to

$$
\operatorname{Vol}_{c}(\mathcal{O}) \leq\left[\Theta^{\prime}: \Theta\right] \cdot \operatorname{Vol}\left(\mathbb{S}^{n}\right)
$$

The required embedding $\Theta \hookrightarrow \Theta^{\prime}$ is easy to obtain for $n=2$ with the index $\left[\Theta^{\prime}: \Theta\right] \leq 2$. Agol checked in his paper that for finite subgroups of $\mathrm{O}(3)$ we have $\left[\Theta^{\prime}: \Theta\right] \leq 4$, and this resolves the case $n=3$. It is also possible to extend this result to a more general class of quasi-reflective groups in dimension $n=3$, which allowed their classification in BM13. In all other cases the classification of finite subgroups of $\mathrm{O}(n)$ is either not known or much more involved, and we do not know how to bound the conformal volume of their quotients. Hence so far we can apply this trick for bounding the conformal volume only in dimensions 2 and 3 .

We now can substitute (4) in (1) and use the bounds for the minimal volume and the spectral gap of the congruence quotients - recall that according to Lemma 2.2 the maximal arithmetic subgroups are always congruence.

In the last section of his paper, Agol indicated the possible quantitative implications of the method, but he was missing some non-trivial technical ingredients required to make it work. It was observed later in Bel09] that one can combine Agol's method with the important technical results of Chinburg and Friedman CF86] in order to obtain the quantitative bounds. It was shown there, in particular, that the degree of the field of definition of arithmetic reflection groups in dimension 3 is bounded above by 35 . In a joint work Belolipetsky and Linowitz BL14 improved this bound to 9, which essentially allows us to give a list of all possible fields of definition (see BL14 for the details). The case of $n=2$ was considered by Maclachlan in [Mac11. Summarizing the results we have:

Theorem 5.3 ([Mac11, BL14]). The fields of definition of arithmetic hyperbolic reflection groups in dimension 2 have degree at most 11, and in dimension 3 at most 9 .

This theorem complements Proposition 5.2 in a stronger form, as it does not impose any additional congruence hypothesis. The cited papers also provide explicit upper bounds for the discriminants of the fields of definition. 


\section{Classification Results in SMall Dimensions}

Consider a quadratic space $(V, f)$, where $V$ is a finite-dimensional vector space over a totally real number field $k$ and $f$ is a non-degenerate quadratic form on $V$. An $\mathfrak{o}_{k}$-module $L$ is called a quadratic lattice if $L$ is a full rank $\mathfrak{o}_{k}$-lattice in $(V, f)$. A quadratic lattice is called even if for the inner product associated with $f$ we have $(v, v) \in 2 \mathfrak{o}_{k}$ for all $v \in L$, and odd otherwise. The dual $L^{*}$ of a quadratic lattice $L$ is the set of all vectors in $V$ having integer inner product with all vectors in $L$. A lattice is called unimodular if $L=L^{*}$, in general, if the inner product is integral on $L$, we have $L \subseteq L^{*}$, and $\Delta(L)=L^{*} / L$ is a finite abelian group. It is called the discriminant group of $L$, and its order is the determinant $\operatorname{det}(L)$. A quadratic lattice $L$ is called strongly square-free if the cardinality of the smallest generating set of $\Delta(L)$ as an $\mathfrak{o}_{k}$-module is at most $\frac{1}{2} \operatorname{rank}(L)$ and every invariant factor of $\Delta(L)$ is square-free. If $k=\mathbb{Q}$ and the inner product associated with $f$ is $\mathbb{Z}$-valued on $L$, we shall call $L$ integral. The level of an integral lattice $L$ is defined to be the minimal positive integer $N$ such that $N(v, v) / 2 \in \mathbb{Z}$ for all $v \in L^{*}$. Integral quadratic lattices of signature $(n, 1)$ or $(1, n)$ (and rank $n+1)$ are called Lorentzian. We refer to [CS99] for more material about quadratic lattices and related structures.

The group $\Gamma_{L}=\operatorname{Aut}(L)$ of the automorphisms of an integral Lorentzian lattice is by definition an arithmetic subgroup of the orthogonal group $\mathrm{O}(n, 1)$. It can be shown that the automorphism group of a non-strongly square-free lattice is always contained in the automorphism group of a strongly square-free one (cf. [All12]).

Lorentzian lattices and their groups of automorphisms arise naturally in $K 3$ surface theory, structure theory of hyperbolic Kac-Moody algebras, and many other fields. The question of their reflectivity was studied by Vinberg, Nikulin, Scharlau, Allcock, and others. There are also some related investigations about reflectivity of lattices defined over quadratic fields. In this section we shall review the classification results which come from this study. Except for an important work of Nikulin on 2-reflective lattices discussed at the end, the other papers deal only with the lattices of small rank.

Nikulin, Allcock, Mark. The case $n=2$ is the first towards the general classification program. Although this case is easier than higher dimensions, the spectral method indicates that it is here that we can expect to encounter the largest number of examples of reflective lattices. In an important paper published in 2000 [Nik00, Nikulin classified the rank 3 reflective strongly square-free Lorentzian lattices. He obtained a list of 1097 lattices which fall into 160 duality classes (a $p$-dual of an integral lattice $L$ is the sublattice of $L^{*}$ corresponding to the $p$-power part of $\Delta(L)$; it can be seen that $L$ and its $p$-dual have the same automorphism group). Note that since every lattice canonically determines a strongly square-free lattice, this classification does contain all integral Lorentzian lattices whose reflection groups are maximal under inclusion. The project was continued more recently by Allcock, who classified all reflective integral Lorentzian lattices of signature $(2,1)$ All12. He showed, in particular, that there are 8595 such lattices which correspond to 374 different reflection groups that fall into 39 commensurability classes. He also checked that the 1097 strongly square-free lattices previously obtained by Nikulin are contained in his list. The method is based on an analysis of the shape of the Coxeter polygons, which allows us to reduce the list of candidates for the reflective lattices to a manageable size, and a subsequent application of Vinberg's algorithm. 
It is worth mentioning that the TeX source file for the Allcock's paper (available from the mathematics e-prints arxiv, see arXiv:1111.1264) can be run as a Perl script that prints out all 8595 lattices with their Gram matrices and other related data in computer-readable format. This brings the results to a form that is suitable for potential applications.

In her PhD thesis Mar15b, Mark studied the classification of rank 3 reflective lattices over quadratic extensions of $\mathbb{Q}$. To this end she adapted the method of Allcock to the real quadratic setting and used a different algorithm for checking reflectivity of a quadratic form termed the walking algorithm. Mark's modifications to Allcock's method are inspired by the previous work of Bugaenko Bug84,Bug90,Bug92. The key difference between the walking and Vinberg's algorithms is the search space: whereas Vinberg's algorithm searches for the new vectors inside an $n$-dimensional polygonal cone, walking has a much more restricted searching area. The main result of Mar15b] is a complete classification of the rank 3 strongly square-free reflective arithmetic hyperbolic lattices defined over $\mathbb{Z}[\sqrt{2}]$. Mark showed that there are 432 such lattices and provided their detailed description, including the structure of the reflection groups. The methods developed in Mar15b] can be applied to the classification problem over other fields, which would be a natural next step of the classification project.

Scharlau-Walhorn. In [SW92, the authors gave two explicit lists of maximal non-cocompact arithmetic reflection groups in dimensions $n=3$ and 4 . The groups are defined by the reflective integral quadratic lattices that are strongly square-free and isotropic. The lists in SW92 contain 49 and 42 lattices, respectively. Later Walhorn found that one example was missing from the list for $n=4$, so there are in total 43 such lattices Wal93. In the notation of [SW92, the 43rd lattice has the shape

$\mathbb{H} \perp\langle 1,7,7\rangle$, where $\mathbb{H}$ is the even unimodular lattice of signature $(1,1)$ and $\langle a, b, c\rangle$ denotes the lattice of the diagonal quadratic form $a x^{2}+b y^{2}+c z^{2}$. It has the determinant $D=-49$ and $r=48$ fundamental roots.

The lattices are shown to be reflective by Vinberg's algorithm, and as a biproduct of the algorithm application the authors also obtained various geometric invariants of the corresponding Coxeter polyhedra, such as the number of faces, the number of cusps, etc. (see also [Sch89, Wal93] for more data). The papers do not provide Coxeter diagrams although they could have been produced from the algorithm output.

The authors indicate how to prove the completeness of the lists (we remark that for $n=3$ they restrict to the isotropic quadratic forms and hence obtain only noncocompact arithmetic reflection groups defined over $\mathbb{Q}$ ); the details of the proof for $n=4$ are given in the dissertation of the second author Wal93].

Belolipetsky-Mcleod. The previous enumeration for $n=3$ is closely related to the study of reflective Bianchi groups. For a square-free positive integer $m$ denote by $O_{m}$ the ring of integers of the imaginary quadratic field $\mathbb{Q}(\sqrt{-m})$. The Bianchi group $\operatorname{Bi}(m)$ is defined by $\operatorname{Bi}(m)=\operatorname{PGL}\left(2, O_{m}\right) \rtimes\langle\tau\rangle$, where $\tau$ acts on $\operatorname{PGL}\left(2, O_{m}\right)$ as complex conjugation. The groups $\operatorname{Bi}(m)$ can be regarded in a natural way as discrete subgroups of the group of isometries of the hyperbolic three-space $\mathbb{H}^{3}$. They are non-cocompact arithmetic subgroups of $\operatorname{Isom}\left(\mathbb{H}^{3}\right)$. One can also define 
the maximal discrete extension of $\operatorname{Bi}(m)$ in $\operatorname{Isom}\left(\mathbb{H}^{3}\right)$, which is called the extended Bianchi group and denoted $\widehat{\mathrm{Bi}}(m)$. Reflectiveness of the Bianchi groups and their extensions was studied by many authors starting from the classical paper of Bianchi Bia91. A trio of papers by Vinberg, Shaiheev, and Shvartsman published all in the same volume Oni87 made an important contribution to the topic by approaching it from the more general perspective of Vinberg's program [Vin90, Sha90, Shv90]. In a related paper Ruz90, Ruzmanoz considered an extended notion of reflectivity called quasi-reflectiveness and gave the first examples of quasi-reflective Bianchi groups. We shall discuss this extension more carefully in Section 9 The research on these topics was concluded in BM13, where we proved the following classification result:

Theorem 6.1. We have

(i) The Bianchi group $\operatorname{Bi}(m)$ is reflective if and only if $m \leq 19, m \neq 14,17$.

(ii) The extended Bianchi group $\widehat{\mathrm{Bi}}(m)$ is reflective if and only if $m \leq 21, m=30$, $33,39$.

(iii) The Bianchi group $\operatorname{Bi}(m)$ is quasi-reflective if and only if $m=14,17,23,31$ and 39.

(iv) The only quasi-reflective extended Bianchi groups are $\widehat{\mathrm{Bi}}(23)$ and $\widehat{\mathrm{Bi}}(31)$.

In the proof, the finite list of candidates was produced using the spectral method and the final step of detecting the reflection groups was again performed by means of Vinberg's algorithm. The paper also provides the Coxeter diagrams and other data for the reflection subgroups. Let us note that the list of reflection groups in BM13 is contained in the Scharlau-Walhorn classification for $n=3$ but does not coincide with it because some integral quadratic forms give rise to non-cocompact arithmetic subgroups commensurable but not contained in Bianchi groups. It is not hard to identify precisely the reflective groups from Theorem 6.1 in the table for $n=3$ in SW92.

In his paper [Sha90], Shaiheev has drawn the schematic pictures of the fundamental polyhedra of the reflection groups that he obtained (we note that there are some small mistakes in Sha90] and refer to BM13 for the corrections). Now, with a complete classification available, it would be good to have a set of computer generated images of the Coxeter polyhedra of these reflection groups. Some nice examples of this type of polyhedra in the upper half-space model of $\mathbb{H}^{3}$ are presented in [JJK $\left.{ }^{+} 15\right]$. Another possible approach is to extend to the non-compact finite volume polyhedra the computer implementation of Andreev's theorem developed by Roeder [Roe07].

Scharlau-Blaschke, Esselmann, Turkalj. In all of the above classification results, the crucial step of determining the reflection subgroup is carried out by means of Vinberg's algorithm. Another approach to classification of the reflective integral lattices is based on the following lemma, which is also due to Vinberg:

Lemma 6.2 (cf. [SW92, Lemma 1.3]). Consider an integral lattice $L=\mathbb{H} \perp M$, where $\mathbb{H}$ is the even unimodular lattice of signature $(1,1)$ and $M$ is positive definite of rank at least 2 . If $L$ is reflective, then the genus $\mathcal{G}(M)$ (which depends only on $L)$ is totally reflective in the sense that every lattice $M^{\prime} \in \mathcal{G}(M)$ is reflective. 
We recall that in the positive definite case a lattice $M$ is called reflective if the reflection subgroup of its automorphisms group has no non-zero fixed vectors in $M \otimes \mathbb{R}$.

It is well known that under quite general conditions an integral lattice $L$ of signature $(n, 1)$ does admit a decomposition of the form $\mathbb{H} \perp M$. In particular, this holds when $n \geq 4$ and $L$ is strongly square-free. Therefore, Lemma 6.2 can be applied for the classification of non-cocompact reflection groups and allows us to reduce the problem to the much better understood positive definite case. Esselmann proved in Ess96] that 20 is the largest dimension for which there exist totally reflective genera. In [SB96], Scharlau and Blaschke used gluing technique to classify positive definite integral reflective lattices in dimensions $\leq 6$. Gluing theory mentioned here provides a method for constructing general integral lattices that contain as a sublattice a direct sum of integral lattices of smaller dimension (see CS99, Chapter 4.3] for the details). In a recent preprint of Turkalj [Tur, the work of Scharlau and Blaschke is combined with other results to give a complete classification of the totally reflective primitive genera in dimensions 3 and 4 , which correspond to the Lorentzian lattices for the hyperbolic dimensions $n=4$ and 5 , respectively. An explicit classification of the square-free totally positive genera for $n=4$ that appeared before in Walhorn's dissertation [Wal93] is reproduced by Turkalj as a subset of the $n=4$ case. The list in Tur contains 1234 genera, of which 289 are square-free and 52 strongly square-free, in dimension 3; and 930 genera, of which 230 are square-free and 88 strongly square-free in dimension 4 . As is expected, the total number of reflective lattices decreases for bigger dimensions.

Nikulin, Vinberg. The case of 2-reflective integral lattices is of a special interest because of its close connection with the theory of $K 3$-surfaces. In particular, the classification of such lattices allows one to describe all algebraic complex surfaces of type $K 3$ whose group of automorphisms is finite. Such a classification is now available thanks to the work of Nikulin and Vinberg.

Recall that an integral lattice $L$ is called 2-reflective if the subgroup of its group of automorphisms generated by 2-reflections, i.e., the reflections whose primitive vectors have square 2, is of finite index. Classification results for the 2-reflective lattices go back to the first papers of Nikulin on the subject; see [Nik79, Nik81b], and Nik84]. These papers cover all the cases except when the rank $r(L)$ is equal to 4. The classification for the latter case was published by Vinberg only in 2007, although he obtained it as early as 1981 Vin07. The methods that are used in Nikulin's papers add algebraic geometry of $K 3$-surfaces to the set of tools that we encountered before.

A complete list of 2-reflective lattices can be found in the above cited papers, here we only reproduce the statistics; see Table 2. The 26 lattices of rank 3 are obtained in [Nik84] (note that the lattices $S_{6,1,2}^{\prime}$ and $S_{6,1,1}$ in the list there are isomorphic), the 14 rank 4 lattices are in [Vin07, and the higher dimensions are treated in Nik81b. For $r \geq 20$ the 2-reflective integral lattices do not exist.

TABLE 2. 2-reflective lattices.

\begin{tabular}{c|ccccccccccccc}
$r(L)$ & 3 & 4 & 5 & 6 & 7 & 8 & 9 & 10 & 11 & 12 & 13 & 14 & $15, \ldots, 19$ \\
\hline \# of lattices & 26 & 14 & 9 & 10 & 9 & 12 & 10 & 9 & 4 & 4 & 3 & 3 & 1
\end{tabular}




\section{EXAMPLES}

Vinberg, Kaplinskaja. Hyperbolic reflection groups in dimensions $n \leq 19$ were found by Vinberg Vin72, and Vinberg and Kaplinskaja VK78. They considered reflection subgroups of the groups of integral automorphisms of the quadratic forms

$$
f\left(x_{0}, x_{1}, \ldots, x_{n}\right)=-x_{0}^{2}+x_{1}^{2}+\cdots+x_{n}^{2} .
$$

For $n=2$ the form was investigated by Lagrange, Gauss, and later by Fricke. In his paper, Fricke showed that the form $-x_{0}^{2}+x_{1}^{2}+x_{2}^{2}$ is reflective and described its Coxeter triangle fundamental domain [Fri91, pp. 64-68] 1 The case $n=3$ first appeared, among other things, in the paper by Coxeter and Whitrow [CW50. For $n \leq 17$ the form was investigated in Vin72, and the remaining $n=18$ and 19 were considered in VK78. The Coxeter polyhedron in dimension 19 is the most complicated one: it has 50 faces and its symmetry group is isomorphic to the symmetric group $S_{5}$. More details can be read from the Coxeter diagrams that are presented in Vin72] and VK78, for each of the cases (see also CS99, Chaper 28]). In [Vin75, Vinberg showed that the form $f$ is not reflective for $n \geq 25$ and indicated that the same should hold for $n \geq 20$ (see also VK78]). The proof of reflectivity in each of the cases is obtained by means of Vinberg's algorithm, while non-reflectivity is shown by detecting infinite-order elements in the quotient $\Gamma / \Gamma_{r}$ of the automorphism group by its reflection subgroup.

In Vin72, Vinberg also investigated the reflection groups of the quadratic forms

$$
f_{2}\left(x_{0}, x_{1}, \ldots, x_{n}\right)=-2 x_{0}^{2}+x_{1}^{2}+\cdots+x_{n}^{2} .
$$

He found that the form is reflective for $n \leq 14$; non-reflectiveness of $f_{2}$ for bigger $n$ is confirmed in Mcleod's thesis [Mcl13, Section 3.1.4]. The Coxeter diagrams for the reflection subgroups in the reflective case are given in Vinberg's paper.

Bugaenko. By the Godement's compactness criterion, for $n \geq 4$ arithmetic groups defined by quadratic forms over $\mathbb{Q}$ are all non-cocompact. Thus in order to see cocompact higher-dimensional examples we have to consider quadratic forms defined over the fields of degree at least 2. This was first done by Bugaenko in 1980s, and his examples still remain essentially the only ones of this type.

In Bug84, Bugaenko investigated the reflection groups of the quadratic forms

$$
f_{\sqrt{5}}\left(x_{0}, x_{1}, \ldots, x_{n}\right)=-\frac{1+\sqrt{5}}{2} x_{0}^{2}+x_{1}^{2}+\cdots+x_{n}^{2} .
$$

He proved that the form is reflective if and only if $n \leq 7$. For $n=8$ he found another admissible quadratic form over the field $\mathbb{Q}(\sqrt{5})$ with discriminant $-(1+\sqrt{5})$ which is reflective Bug92. This is the highest dimension for which we know examples of cocompact hyperbolic reflection groups. The Coxeter diagrams for the reflection polyhedra are presented in Bug84 and Bug92.

In another article Bug90, Bugaenko considered the quadratic from

$$
f_{\sqrt{2}}\left(x_{0}, x_{1}, \ldots, x_{n}\right)=-(1+\sqrt{2}) x_{0}^{2}+x_{1}^{2}+\cdots+x_{n}^{2},
$$

which are shown to be reflective if and only if $n \leq 6$. The Coxeter diagrams for $n \leq 5$ are given in the paper (note that while there are some minor typographical errors in the node-labeling for $n=4$ and 5 , the vectors computed by the algorithm

\footnotetext{
${ }^{1}$ We thank John Ratcliffe for suggesting this reference.
} 
are correct), and the data related to $n=6$ provided by Bugaenko can be found in All06, Tabels 2.1, 2.2].

Reflectivity of the quadratic forms in each of the cases is checked by a variant of Vinberg's algorithm, with the modifications that make it work over the algebraic integers. To show non-reflectivity, Bugaenko systematically used a criterion of detecting an infinite-order symmetry of the Coxeter polyhedron associated to a loxodromic isometry of $\mathbb{H}^{n}$.

Several other examples of cocompact arithmetic hyperbolic reflection groups similar to the ones that were considered by Bugaenko were found in Mcleod's thesis Mcl13. The case $n=2$ over $\mathbb{Q}(\sqrt{2})$ was thoroughly studied by Mark (see the previous section). In [All13, Section 4], Allcock obtained an example of a cocompact reflection group in $\mathbb{H}^{7}$ from the reflection centralizer in Bugaenko's example in $\mathbb{H}^{8}$. This trick can be repeated to get an even more complicated example in $\mathbb{H}^{6}$. It would be interesting to know whether or not the resulting groups are commensurable with Bugaenko's examples.

Allcock, Potyagailo-Vinberg. In All06, Allcock proved that there exist infinitely many finite-covolume (resp. cocompact) arithmetic hyperbolic reflection groups acting on hyperbolic space $\mathbb{H}^{n}$ for every $n \leq 19$ (resp. $n \leq 6$ ). This implies, in particular, that the maximality assumption in the finiteness Theorem 4.3 cannot be dropped. The construction is based on examples of Vinberg, VinbergKaplinskaja, and Bugaenko described above and a simple redoubling trick:

Call a wall of a Coxeter polyhedron $\mathrm{P}$ a doubling wall if the angles it makes with the walls it meets are all even submultiples of $\pi$. By the double of $\mathrm{P}$ across one of its walls we mean the union of $\mathrm{P}$ and its image under reflection across the wall. A polyhedron is called redoublable if it is a Coxeter polyhedron with two doubling walls that do not meet each other in $\mathbb{H}^{n}$. It is easy to show that the double of a Coxeter polyhedron $\mathrm{P}$ across a doubling wall is itself a Coxeter polyhedron. Moreover, if the doubling wall is disjoint from another doubling wall so that $\mathrm{P}$ is redoublable, then the double is also redoublable. This allows one to iterate the procedure and produce an infinite series of finite volume Coxeter polyhedra.

The simplest redoublable polyhedra are the right-angled polyhedra, they have all the dihedral angles equal to $\pi / 2$. These polyhedra were studied by Potyagailo and Vinberg in PV05, who showed that they may exist only for $n \leq 4$ in the compact case and for $n \leq 14$ in the general finite-volume case. Using a similar method, the second bound was later improved by Dufour to $n \leq 12$ [Duf10]. Examples of compact right-angled polyhedra in $\mathbb{H}^{n}$ are known for all $n \leq 4$ and finite-volume ones only for $n \leq 8$ (see [PV05]). For the other dimensions Allcock showed that many of the arithmetic examples discussed above are redoublable. It is worth mentioning that there also exist non-redoublable Coxeter polyhedra, the simplest example shown to the author by Daniel Allcock is the hyperbolic triangle with all angles equal $\frac{\pi}{7}$ - it is easy to check that the group generated by reflections in its sides does not have any finite index reflection subgroups except itself. It would be interesting to check if the same phenomenon occurs for the Borcherds polyhedron in $\mathbb{H}^{21}$, which is discussed below.

Allcock mentions that his method resembles Ruzmanov's construction of nonarithmetic Coxeter polyhedra from Ruz89. The latter was recently further elaborated by Vinberg to produce some new examples of non-arithmetic hyperbolic reflection groups Vin14. 
Mcleod, Mark. The spectral method indicates that we should look for examples of arithmetic reflection subgroups in arithmetic lattices of small covolume. Recall that for every dimension the covolume of lattices in $\operatorname{Isom}\left(\mathbb{H}^{n}\right)$ is uniformly bounded from below (by the Kazhdan-Margulis theorem [KM68]), and the precise minimal value in the arithmetic case is known Bel04, Bel07, BE12. For most of the dimensions the minimum is attained on the arithmetic subgroups associated to the quadratic form $f$ considered by Vinberg, but quite surprisingly, for $n=4 k-1 \geq 7$, it corresponds to

$$
f_{3}\left(x_{0}, x_{1}, \ldots, x_{n}\right)=-3 x_{0}^{2}+x_{1}^{2}+\cdots+x_{n}^{2} .
$$

Reflection groups of these quadratic forms were investigated by Mcleod in [Mcl11]. He showed that $f_{3}$ is reflective for $n \leq 13$ and non-reflective for bigger $n$. The proofs use Vinberg's algorithm and some results of Bugaenko. Similar to the previous cases, arguably the most interesting example appears in the highest dimension $n=$ 13 - its Coxeter polyhedron has 22 faces and the group of symmetries isomorphic to $\mathbb{Z}_{2} \times \mathbb{Z}_{2}$. The Coxeter diagrams are given in Mcleod's paper.

The next natural step in this direction is to investigate the quadratic forms

$$
f_{m}\left(x_{0}, x_{1}, \ldots, x_{n}\right)=-m x_{0}^{2}+x_{1}^{2}+\cdots+x_{n}^{2} .
$$

This was done by Mark for the case $m=p$, a prime number Mar15a. She showed that:

$f_{5}$ is reflective for $2 \leq n \leq 8$;

$f_{7}$ and $f_{17}$ are reflective for $n=2$ and 3 ;

$f_{11}$ is reflective for $n=2,3$, and 4 .

She also proved that for other $p$ and in higher dimensions $f_{p}$ is non-reflective. Together with some Nikulin's results for $n=2$ this gives a complete list of the reflective forms of this type. Related results were also obtained by Mcleod in his thesis Mcl13] in particular, he gave a complete list of the reflective quadratic forms $f_{m}$ for all natural $m$ in all dimensions (see [Mcl13, Table 3.1, page 37]).

Borcherds. In Bor87, Borcherds found an example of a non-cocompact arithmetic reflection group in $\mathbb{H}^{21}$, which was later shown by Esselmann Ess96] to have the largest possible dimension. Borcherds started from Conway's description of the group of automorphisms of the even unimodular Lorentzian lattice of rank 26 in Con83: it is a semidirect product of the reflection subgroup (of infinite index) and the group of affine automorphisms of the famous Leech lattice. We shall come back to this group in the next section. The finite-volume Coxeter polyhedron $\mathrm{P}^{21}$ in $\mathbb{H}^{21}$ comes out as a face corresponding to the spherical diagram of type $\mathrm{D}_{4}$ of the infinite-volume 25-dimensional Conway's polyhedron. A general method of determining the shape of a face of a Coxeter polyhedron motivated by this and other examples is described in All06.

The polyhedron $\mathrm{P}^{21}$ can also be obtained using Vinberg's algorithm. Following Borcherds, its group is the reflection subgroup of $\mathrm{O}_{0}(g, \mathbb{Z})$, where $g$ is a quadratic form of signature $(21,1)$ associated to the even sublattice $L$ of $\mathbb{Z}^{21,1}$ (i.e., $L$ consists of the integral vectors with the even sum of the coordinates). We can take the controlling vector $u_{0}=v_{0}$, the first basis vector (assuming $\left(v_{0}, v_{0}\right)=-1$ ). Its stabilizer is generated by reflections corresponding to the remaining 21 basis vectors, and it is a finite Coxeter group of type $\mathrm{D}_{21}$. The next vector produced by the algorithm is $e_{22}=v_{0}+v_{1}+v_{2}+v_{3}$, etc. The polyhedron $\mathrm{P}^{21}$ has 210 sides with 42 of them corresponding to the 2-reflections, and the remaining 168 to the 4-refections 
in $\mathrm{O}_{0}(g, \mathbb{Z})$. It has a very large symmetry group isomorphic to $\operatorname{PSL}\left(3, \mathbb{F}_{4}\right) \cdot D_{6}$ of order 241920 (here $D_{6}$ denotes the dihedral group of order 12). It would interesting to try to draw its Coxeter diagram in a maximally symmetric way.

Daniel Allcock showed me a nice way to view the diagram of $\mathrm{P}^{21}$ on the projective plane $\mathbb{F}_{4} \mathrm{P}^{2}$ over the field $\mathbb{F}_{4}$ with four elements: One can index the faces of $\mathrm{P}^{21}$ by the 21 points, 21 lines, and 168 hyperconics in $\mathbb{F}_{4} \mathrm{P}^{2}$. The edges of the diagram are determined by the incidence relations. The resulting structure is invariant under the full group of automorphisms of $\mathbb{F}_{4} \mathrm{P}^{2}$ (including Galois conjugation and the point-line interchange), altogether producing the full group of symmetries of $\mathrm{P}^{21}$. A cute algebraic geometric application of the Borcherds group related to this viewpoint was found by Dolgachev and Kondo DK03. They constructed a unique super-singular $K 3$ surface in characteristic 2 satisfying a set of equivalent properties whose automorphism group is the symmetry group of an infinite treelike polyhedron obtained by gluing together the copies of $\mathrm{P}^{21}$.

The 21-dimensional polyhedron considered above is a very special and quite complicated object, but the most complicated currently known finite-volume example lives a few dimensions below. It was also discovered by Borcherds, but in a different paper [Bor00] and using a very different method. The idea of [Bor00] is that many interesting reflection groups (in particular, most of the known examples in dimensions at least 5) can be obtained from reflective singularities at cusps of modular forms of $\mathrm{SL}(2, \mathbb{Z})$. This way Borcherds found new examples of arithmetic reflection groups without a priori writing down any roots and reflections! We shall review this method in the next section. The most complicated new example in $\mathbb{H}^{n}$ is described in [Bor00, p. 346]: it is a 17-dimensional non-compact finite-volume polyhedron with 960 sides. Very little is currently known about geometry of this polyhedron.

Other examples. We conclude the discussion of classification and examples by mentioning briefly some other results. There is another natural approach to the classification problem for hyperbolic reflection groups that, rather than looking at the admissible quadratic forms and lattices in $\operatorname{Isom}\left(\mathbb{H}^{n}\right)$, begins with analyzing the possible shapes of the Coxeter polyhedra in $\mathbb{H}^{n}$. The first class of polyhedra that comes out here consists of hyperbolic Coxeter simplices. Their study goes back to the work of Coxeter and Lannér in the first half of the twentieth century. In 1950, Lannér enumerated bounded hyperbolic Coxeter simplices and showed that they exist only in dimensions $n \leq 4$ [Lan50]. Later the enumeration was extended to the unbounded Coxeter simpleces of finite volume that exist in dimensions $n \leq 9$. More recently, Johnson, Kellerhals, Ratcliffe, and Tschantz described the commensurability classes of the hyperbolic Coxeter simplex reflection groups in all the dimensions $9 \geq n \geq 3$ [JKRT02]. They also showed that for $n \geq 4$, all of these groups except for one five-dimensional example are arithmetic. In a series of papers Felikson and Tumarkin studied other types of the hyperbolic Coxeter polyhedra (without connection to arithmeticity). We refer to [FT14 and the references therein for related results.

\section{REFLECTIVE MODULAR FORMS}

Let $\Gamma$ be a lattice in $\mathrm{SL}(2, \mathbb{R})$. A modular form of weight $k$ with respect to $\Gamma$ is a complex-valued function $f$ on the hyperbolic plane $\mathbb{H}=\{z \in \mathbb{C} \mid \operatorname{Im}(z)>0\}$ in 
the upper half-plane model which is holomorphic on $\mathbb{H}$, holomorphic at the cusps of $\Gamma$, and satisfies the equation

$$
f\left(\frac{a z+b}{c z+d}\right)=(c z+d)^{k} f(z), \text { for all } z \in \mathbb{H} \text { and }\left(\begin{array}{ll}
a & b \\
c & d
\end{array}\right) \in \Gamma .
$$

A modular form is called a cusp form if it vanishes at the cusps of $\Gamma$. For example, if $\Gamma=\mathrm{SL}(2, \mathbb{Z})$, this condition means that $f(z) \rightarrow 0$ when $z \rightarrow i \infty$.

The theta (or Howe) correspondence assigns to a cusp form $f$ a cuspidal automorphic form $\phi_{f}$ on the bounded symmetric domain associated with a group $\mathrm{O}(m, n)$. We are interested in the singular theta correspondence, which allows $f$ to have poles at the cusps and as the output produces a meromorphic modular form $\phi_{f}$. It can be shown then that under certain conditions on $f$ the singularities of $\phi_{f}$ are reflection hyperplanes of an arithmetic reflection group or a quasi-reflection group. We shall proceed with a more precise description of the correspondence.

Let $L$ be an integral lattice of signature $(m, n)$ and $\operatorname{Gr}(L)$ denote the Grassmannian of the maximal ( $m$-dimensional) positive definite subspaces of $L \otimes \mathbb{R}$. It is a symmetric space of dimension $m n$ acted upon by the orthogonal group $\mathrm{O}(m, n)$. We note that in this section the Lorentzian lattices have signature $(1, n)$, which gives opposite signs of inner products compared to the rest of the paper. We decided not to change the notation in order to comply with the literature. Given an element $v \in \operatorname{Gr}(L)$ and $\lambda \in L \otimes \mathbb{R}$, we denote by $\lambda_{v^{+}}$and $\lambda_{v^{-}}$the projections of $\lambda$ onto the positive definite space represented by $v$ and the negative definite space orthogonal to $v$.

Suppose the lattice $L$ is even. The Siegel theta function of a coset $L+\gamma$ of $L$ in $L^{*}$ is

$$
\Theta_{L+\gamma}(\tau ; v)=\sum_{\lambda \in L+\gamma} \exp \left(\pi i \tau \lambda_{v^{+}}^{2}+\pi i \bar{\tau} \lambda_{v^{-}}^{2}\right),
$$

where $\tau \in \mathbb{H}$ and $v \in \operatorname{Gr}(L)$. Combining these for all elements of $L^{*} / L$ gives a $\mathbb{C}\left[L^{*} / L\right]$-valued function called the Siegel theta function of $L$,

$$
\Theta_{L}(\tau ; v)=\sum_{v \in L / L^{*}} e_{\gamma} \Theta_{L+v}(\tau ; v),
$$

where $e_{\gamma}$ denotes the elements of the standard basis of the group ring $\mathbb{C}\left[L^{*} / L\right]$.

When dealing with theta functions of lattices, half-integral weight vector-valued modular forms naturally occur. They can be defined using the metaplectic double cover $\widetilde{\mathrm{SL}}(2, \mathbb{R})$. We refer to Bor98 for the details of this construction. The Siegel theta function $\Theta_{L}(\tau ; v)$ is a vector-valued modular form of weight $(m / 2, n / 2)$ and type $\rho_{L}$, where $\rho_{L}$ is the Weil representation of the group $\widetilde{\mathrm{SL}}(2, \mathbb{Z})$ on the vector space $\mathbb{C}\left[L^{*} / L\right]$.

Let $F$ be another vector-valued modular form which has weight $(-m / 2,-n / 2)$ and type $\rho_{L}$. Then the product $F(\tau) \overline{\Theta(\tau ; v)}$ is a modular form of weight 0 . If this product is of sufficiently rapid decay at $i \infty$ (which occurs if $F$ is a cusp form), we can take the integral

$$
\Phi_{F}(v)=\int_{\mathcal{F}} F(v) \overline{\Theta(\tau ; v)} \frac{d x d y}{y^{2}}
$$

where $\mathcal{F}=\{\tau \in \mathbb{H}|| \tau|\geq 1,| \operatorname{Re}(\tau)|\leq 1 / 2|\}$ is the usual fundamental domain for $\operatorname{SL}(2, \mathbb{Z})$. This gives us a function $\Phi_{F}$ on $\operatorname{Gr}(L)$ invariant under a congruence 
subgroup of $\operatorname{Aut}(L)$. The map $F(\tau) \rightarrow \Theta_{F}(v)$ is essentially the original theta correspondence.

Suppose now that we allow $F(\tau)$ to have singularities at the cusps but require it to be holomorphic on $\mathbb{H}$. The integral above diverges wildly. Harvey and Moore used ideas from quantum field theory to show that it is still possible to make sense of the integral by regularization HM96. Their construction was further generalized by Borcherds in Bor98. The idea of regularization is to truncate the integration domain in such a way that most of the wildly non-convergent terms vanish. The remaining non-convergent terms are of polynomial growth and can be dealt with easily. The truncated domains are

$$
\mathcal{F}_{t}=\{\tau \in \mathbb{H}|| \tau|\geq 1,| \operatorname{Re}(\tau)|\leq 1 / 2|, \operatorname{Im}(\tau) \leq t\}
$$

The regularized value of the integral is defined as the value at $r=0$ of the analytic continuation of

$$
\lim _{t \rightarrow \infty} \int_{\mathcal{F}_{t}} F(\tau) \overline{\Theta(\tau ; v)} \frac{d x d y}{y^{2+r}}
$$

This defines a more general map $F(\tau) \rightarrow \Phi_{F}(v)$, which is called the singular theta correspondence. It is easy to check that the singularities of $\Phi_{F}(v)$ occur on subGrassmannians of the form $v^{\perp}$ for $v \in L^{*},(v \cdot v)<0$, where there is a non-zero coefficient corresponding to $v$ in the Fourier expansion of $F$ at the cusp. If the singularities occur along the reflection hyperplanes of the underlying lattice $L$, we shall call the modular form $F(\tau)$ a reflective modular form. Not all reflective lattices correspond to reflective modular forms, but many particularly interesting examples do have this property.

The construction can be generalized to modular forms of the groups $\Gamma$ different from $\operatorname{SL}(2, \mathbb{Z})$; moreover, it is often possible to use scalar-valued modular forms of level $N$ instead of the vector-valued modular forms. We refer to Bor00] for the details. A useful sufficient condition for a modular form to be reflective is given in Bor00, Lemma 11.2]. For example, if $N$ is a square-free integer and $\Gamma=\Gamma_{0}(N)=$ $\left\{\left(\begin{array}{ll}a & b \\ c & d\end{array}\right) \in \mathrm{SL}(2, \mathbb{Z}) \mid c \equiv 0 \bmod N\right\}$ is a congruence subgroup, a modular form $F(\tau)$ for $\Gamma$ of weight $\frac{m-n}{2}$ is reflective for an even lattice $L$ of signature $(m, n)$ and level $N$ if the poles of $F(\tau)$ at all cusps of $\Gamma$ are simple.

We conclude this section with some examples of reflective modular forms from Bor00.

Example 8.1. The first case to consider is $N=1, \Gamma=\operatorname{SL}(2, \mathbb{Z})$ and $L$ is an even integral lattice of signature $(m, n)$ and level 1 . It is well known that the modular forms of $\Gamma$ form a polynomial ring generated by the Eisenstein series $E_{4}(\tau)=1+240 q+2160 q^{2}+\cdots$ and $E_{6}(\tau)=1-504 q-16632 q^{2}-\cdots$, with $q=e^{2 \pi i \tau}$, of weights 4 and 6 , respectively. The dimensions of the spaces of modular forms of different weights are given by the coefficients of the Hilbert function $1 /\left(1-x^{4}\right)\left(1-x^{6}\right)$. We refer to Miy89 for these and other related facts from the classical theory of modular forms. The first weight in which we have a non-trivial modular form is $k=12$, and the critical form is $\Delta(\tau)=\eta(\tau)^{24}=q \prod_{k>0}\left(1-q^{k}\right)^{24}$. The forms $f=E_{4}(\tau)^{k} / \Delta(\tau)$ have simple poles at the cusp of $\Gamma$ at $i \infty$. It follows that they are reflective modular forms for even lattices $L$ of level $N=1$ and signature $m-n \geq-24, m-n=0 \bmod 8$. 
Here are some concrete cases:

For $m-n=-24$, we can take $f=1 / \Delta(\tau)=q^{-1}+24+324 q+\cdots$ of weight -12 and a simple pole at the cusp. Two examples arising here are of a special interest: in the Lorentzian case we have a lattice $L=I I_{1,25}$, which is a quasi-reflective lattice first discovered by Conway Con83] in signature $(2,26)$ the reflective lattice $I I_{2,26}$ plays an important role in the arithmetic mirror symmetry studied by Gritsenko and Nikulin.

For $m-n=-16$, we take $f=E_{4}(\tau) / \Delta(\tau)=q^{-1}+264+8244 q+\cdots$ of weight -8 and find the reflective Lorentzian lattice $I I_{1,17}$. Similarly, for $m-n=-8$ with $f=E_{4}(\tau)^{2} / \Delta(\tau)=q^{-1}+504+73764 q+\cdots$ of weight -4 , we obtain the reflective lattice $I I_{1,9}$. The arithmetic reflection groups associated with these lattices were first described by Vinberg in Vin75.

Example 8.2. Suppose the level $N=2$. The group $\Gamma=\Gamma_{0}(N)$ has two cusps which can be taken as $i \infty$ and 0 . The ring of modular forms for $\Gamma$ is a polynomial ring on generators $-E_{2}(\tau)+2 E_{2}(2 \tau)=1+24 q+24 q^{2}+\cdots$ of weight 2 and $E_{4}(\tau)$ of weight 4 . The Hilbert function is $1 /\left(1-x^{2}\right)\left(1-x^{4}\right)$.

As $N$ is square-free, all poles of order at most 1 are reflective by Lemma 11.2 of Bor00. There are also other possible reflective singularities but we will not consider them here. By looking at the form $\Delta_{2+}(\tau)^{-1}=\eta(\tau)^{-8} \eta(2 \tau)^{-8}$ of weight -8 with simple poles at the cusps, we see that all level 2 even lattices of signature at least -16 have reflective modular forms. The Lorentzian lattices $I I_{1,17}\left(2^{+8}\right)$ and $I I_{1,17}\left(2^{+10}\right)$ are quasi-reflective as was the case for the lattice $I I_{1,25}$ in the previous example (we refer to [CS99] for the notation used here). The next example $L=I I_{1,17}\left(2^{+6}\right)$ gives us the 17-dimensional arithmetic reflection group discovered by Borcherds in [Bor00]. This example was mentioned at the end of the previous section.

\section{More about the Structure of the Reflective Quotient}

Let $\Gamma_{0}<\operatorname{Isom}\left(\mathbb{H}^{n}\right)$ be an arithmetic subgroup, let $\Gamma \unlhd \Gamma_{0}$ be its maximal subgroup generated by reflections in hyperplanes, and let $\Theta=\Gamma_{0} / \Gamma$ be the reflective quotient. We have the following possibilities for the group $\Theta$ :

(a) finite group;

(b) affine crystallographic group;

(c) non-amenable group.

In case (a) the group $\Gamma$ is an arithmetic reflection group, while in (b) and (c) it has infinite covolume and hence is not a lattice. Case (b) is known as quasi-reflective or parabolic-reflective. The second term refers to the fact that in this case the group $\Theta$ is virtually isomorphic to an affine group generated by parabolic transformations of $\mathbb{H}^{n}$. Recall that a discrete group is called amenable if it has a finitely additive left-invariant probability measure. Finite groups and affine crystallographic groups are amenable; hence the first two cases can be joined together into the amenable type. This type is relatively rare, and the known results imply that generically the reflective quotients are non-amenable.

Note that by the Tits alternative these are the only possible cases. Indeed, the group $\Theta$ is a finitely generated linear group, hence by Tit72 it is either virtually solvable or contains a non-abelian free subgroup. A virtually solvable group acting discretely on hyperbolic space (recall that by Lemma 2.3, the group $\Theta$ is isomorphic 
to the group of symmetries of the Coxeter polyhedron of $\Gamma$ ) is virtually free abelian, which brings us to a one of the first two cases. On the other hand, it is well known that the groups that contain non-abelian free subgroups are non-amenable. It is worth pointing out that case (b) can happen only if $\Gamma_{0}$ is not cocompact, since in cocompact case we have a simple alternative that either the reflective quotient $\Theta$ is finite or it is non-amenable.

Most of this survey is dedicated to the reflective groups (a). The first and arguably the most interesting example of a quasi-reflective group was discovered by Conway in Con83. He showed that for the group of automorphisms of the 26dimensional even unimodular Lorentzian lattice its reflective quotient is isomorphic to the group of affine automorphisms of the Leech lattice. Therefore, we have an example of a quasi-reflective arithmetic group in hyperbolic dimension $n=$ 25. The proof in Conway's paper is very short but it relies on many results from the previous study of the Leech lattice by Conway and others. It is conjectured that $n=25$ is the largest dimension where there exists a quasi-reflective group. This conjecture can be possibly resolved by Esselmann's method [Ess96, which he applied to find the maximal dimension of isotropic reflective lattices, but such a proof is not available so far. In his doctoral dissertation Bar03, Barnard showed that the conjecture can be deduced from an open conjecture of Burger, Li and Sarnak about the automorphic spectra of orthogonal groups BS91, BLS92, It is interesting to note that another conjecture considered in [BS91] has already appeared in this survey while we were discussing the spectral method, and Barnard's approach is based on reflective modular forms and it highlights yet another relation between arithmetic reflection groups and the spectrum of the Laplacian.

In Nik96, Nikulin proved using his method that in any dimension $n$ there exists only finitely many maximal arithmetic hyperbolic quasi-reflective groups (see Theorem 1.1.3 ibid.). He mentions that it is not difficult to show that there are not any such groups for $n \geq 43$, and he also conjectures that the sharp bound should be given by the Conway's example. It would be interesting to find a spectral proof for the finiteness theorem, which may potentially give better quantitative bounds for these groups in a fixed dimension. The only result of this kind available so far can be found in [BM13, Section 5], where we used Agol's trick (cf. Section 5) and the classification of the plane crystallographic groups to obtain a good quantitative bound for the maximal quasi-reflective groups in dimension $n=3$. Note that similar to the reflective case, the maximality assumption is essential: an example of an infinite sequence of arithmetic quasi-reflective groups in dimension 2 is given in Nik96, Example 1.3.4].

Following Conway, quasi-reflective groups related to the Leech lattice were studied by Borcherds who, in particular, found several other examples in smaller dimensions (see Bor90, Theorem 3.3]). Later examples of quasi-reflective groups were constructed using quasi-reflective modular forms Bor00, Bar03. In Ruz90, Ruzmanov considered quasi-reflective groups in dimension 3 from the geometric viewpoint. He introduced the notion of a quasi-bounded Coxeter polyhedron and found examples of quasi-reflective Bianchi groups. This research was concluded in BM13, where all the quasi-reflective Bianchi groups and extended Bianchi groups are classified (cf. Theorem 6.1(iii, iv)).

\footnotetext{
${ }^{2}$ We thank Richard Borcherds for mentioning this important result.
} 
By the classification of the possible types of the reflective quotients, the upper bounds for the dimension of the reflective and quasi-reflective groups imply the lower bound for the dimension in which the quotient is necessarily non-amenable. A different approach to the problem was undertaken by Meiri in Mei14, who directly constructed non-abelian free subgroups of the reflective quotients in sufficiently large dimension for the forms defined over $\mathbb{Q}$. A part of Meiri's argument is closely related to the proof of non-reflectivity of the high-dimensional quadratic forms in Vin84a. Unfortunately, the quantitative bounds on the dimension obtained in Mei14 are far from sharp.

It would be interesting to know what we can say about the reflective quotient group when it is non-amenable: Is it (relatively) hyperbolic? CAT(0)? Does it have a uniformly bounded spectral gap?, etc.

\section{Open PROBLEMS}

Generalizations. In the very beginning of the paper we discussed Petrunin's question [MOv12], and we recall it again here:

Problem 10.1. Do there exist any hyperbolic lattices in the spaces of large dimension which are generated by elements of finite order?

As before, the question can be restricted to the arithmetic hyperbolic lattices. The answer is unknown in both cases, but we can expect it to be negative.

A related problem appears in a recent paper by Fuchs, Meiri, and Sarnak (cf. [FMS14, page 1621]):

Problem 10.2. Are there any hyperbolic lattices generated by reflections and Cartan involutions (also called "reflections in points") in the hyperbolic spaces of sufficiently large dimension?

A negative answer to this question would allow us to settle Conjecture 2 in FMS14. An example of a lattice generated by Cartan involutions in $\mathbb{H}^{8}$ can be found in All99, Theorem 5.3]. This problem is, of course, a very special case of Problem 10.1 .

The groups of isometries of the hyperbolic $n$-space has real rank 1 . It is worth mentioning that for the irreducible lattices in higher real rank semisimple Lie groups $H$ the situation is very different. We can consider a lattice $\Gamma_{0}$ in such a group $H$ which has a non-central element $g$ of finite order. Let $\Gamma$ be the normal subgroup of $\Gamma_{0}$ generated by all the conjugates of $g$. By the Margulis normal subgroup theorem (see [Mar91, Chapter IV]), the group $\Gamma$ is then itself a lattice in $H$. We can leave it as an exercise for the reader to check that it is generated by a finite set of $g$ conjugates. Hence we can easily produce various examples of lattices in such groups $H$ which are generated by elements of finite order.

Basic examples of this kind of lattices in higher rank groups come from the orthogonal groups $\mathrm{O}(n, m), n, m \geq 2$. Similar to the case of the hyperbolic $n$-space (corresponding to $m=1$ ), we can consider here lattices generated by reflections. For instance, for the quadratic form

$$
f_{n, m}\left(x_{1}, x_{2}, \ldots, x_{n+m}\right)=-x_{1}^{2}-\cdots-x_{m}^{2}+x_{m+1}^{2}+\cdots+x_{m+n}^{2},
$$

the group generated by reflections will be an arithmetic lattice in $\mathrm{O}(n, m)$ according to the arithmeticity and normal subgroup theorems of Margulis Mar91. These lattices, however, will never be Coxeter groups because as lattices in a higher rank 
Lie group, they have Kazhdan's property (T) [Kaz67, while on the other hand the infinite Coxeter groups are known not to have Kazhdan's property BJS88. It would be good to know more about the algebraic structure of these groups:

Problem 10.3. Obtain examples of presentations for lattices in $\mathrm{O}(n, m), n, m \geq 2$, generated by reflections or, more generally, for irreducible lattices in higher rank semisimple Lie groups generated by elements of finite order.

Considering the infiniteness of the family of higher rank reflection groups, the natural question is which of them are really interesting. In Bor00, Borcherds suggested that interesting reflective lattices should be associated to reflective modular forms and gave examples of such lattices (cf. Section 8). In a recent preprint Ma15, Ma studied the basic class of 2-reflective modular forms proving that there are only finitely many 2-reflective lattices of signature $(2, n)$ with $n \geq 7$ and there are no such lattices when $n \geq 30$. Here a lattice $L$ is called 2-reflective if the subgroup of its group of automorphisms generated by -2-reflections is of finite index and $L$ is associated with a reflective modular form. Clearly, the second condition is crucial for the finiteness result. The largest $n$ for which we know an example of this kind is $n=26$ [Bor00, p. 344] with the corresponding lattice $L=I I_{2,26}$ (cf. Example 8.1).

Towards classification. There are two main problems that appear on the way towards classification of arithmetic hyperbolic reflection groups:

Problem 10.4. Find good bounds for the arithmetic invariants of the reflective quadratic forms in arbitrary dimension.

Problem 10.5. Check reflectivity of a given quadratic form.

The quantitative bounds that can be extracted from the proofs of the finiteness theorems in ABSW08, or Nik07] are huge and have no practical value. In Section 5 we explained how Problem 10.4 can be solved under a certain additional arithmetic assumption (requiring that the maximal reflection groups are congruence) or for dimensions $n \leq 3$. One can try to push the technique from the low dimensions to higher $n$, or try to investigate what kind of limitations are in fact implied by the congruence assumption. We can also produce the conditional list and use it as a heuristic for generating all the compatible examples. It is plausible that this list would actually cover all higher-dimensional examples. For sufficiently large $n$, say $n \geq 10$, the conditional list of the candidates would be quite small. Another interesting direction is to try to find all lattices that are associated to reflective modular forms. Is there any connection between these lattices and the congruence reflection groups?

The main tool for checking reflectivity of a quadratic form is Vinberg's algorithm discussed above. There are several computer implementations of this algorithm but none of them is publicly available or standard. The case when the ring of integers $\mathfrak{o}_{k}$ of the defining field is not a PID requires a special attention as in this case the transformations from $\Gamma$ are not necessarily given by the matrices with $\mathfrak{o}_{k}$-entries. We do not know any examples of arithmetic reflection groups that would highlight this issue, but they may exist, in particular, in the spaces of small dimension.

About examples. Geometry of the high-dimensional hyperbolic Coxeter polyhedra remains mysterious in many ways. This refers also to the known examples of such polyhedra. Here we can state the following problem: 
Problem 10.6. Investigate geometrical properties and the combinatorial structure of high-dimensional hyperbolic Coxeter polyhedra.

In this statement the high dimension may refer to $n \geq 6$ in the compact case and to $n \geq 10$ for the finite-volume non-compact hyperbolic polyhedra. There are only a handful of known examples in these dimensions (cf. Section 7). Which of them are redoublable or mixable in the sense of [All06] and Vin14], respectively? What are the covering/comensurability relations between the higher-dimensional examples? How many faces, vertices, and cusps do these polyhedra have? These numbers are known for most of the examples, but are there any interesting relations between them beyond the ones that we already know?

A hyperbolic version of the Cartan-Killing classification. Daniel Allcock raised this problem. A fundamentally important feature of the finite and affine Coxeter groups is their connection to Lie theory. This connection extends to hyperbolic Coxeter groups and Kac-Moody theory. In a series of papers Gritsenko and Nikulin isolated the key properties of Borcherds' fake monster Lie algebra and stated the corresponding classification problem for the class of the generalized Kac-Moody algebras which they call the Lorentzian Kac-Moody algebras (see GN02]).

The classification problem can be stated in terms of root systems. Let us call a set $\Pi$ of spacelike (positive-norm) vectors in $\mathbb{E}^{n, 1}$ a simple root system if $\left(v, v^{\prime}\right)$ is non-positive and lies in $\frac{1}{2}(v, v) \mathbb{Z}$, for all $v, v^{\prime} \in \Pi$. The integral $\operatorname{span} L$ of the simple roots is called the root lattice. The Weyl group $W$ means the group generated by the reflections in the roots. if:

A simple root system $\Pi$ spanning $\mathbb{E}^{n, 1}$ satisfies the Gritsenko-Nikulin conditions

(i) there exists a Weyl vector $\rho \in \mathbb{E}^{n, 1}$ such that $(v, \rho)=-(v, v) / 2$ for all $v \in \Pi$;

(ii) the normalizer of $W$ has a finite index in the orthogonal group $\mathrm{O}(L)$;

(iii) the group $W$ has the arithmetic type (cf. [Nik96, Section 1.4] or [All15, p. 326-327] for several equivalent definitions of this notion).

In the terminology of [Nik96] such root systems are said to have restricted arithmetic type. Under these conditions, it turns out that the Weyl vector $\rho$ is unique and must be timelike or lightlike (i.e., $(\rho, \rho)<0$ or $=0$, respectively). In the timelike case, the (projectivized) Weyl chamber is a finite-volume polyhedron in hyperbolic space, while in the lightlike case, the Weyl chamber has infinite volume and infinitely many sides.

Problem 10.7. Classify the simple root systems of rank at least 3 that satisfy the Gritsenko-Nikulin conditions and corresponding Lorentzian Kac-Moody algebras.

This problem is closely related to the classification problem for the Lorentzian reflective and quasi-reflective lattices. The main difference is the existence of the Weyl vector $\rho$ which is related to the automorphic features of the Lorentzian algebras (recall, in particular, the Borcherds reflective modular form mentioned in Section (7). The known results about hyperbolic reflection groups allowed Nikulin to prove finiteness theorems for the Lorentzian Kac-Moody algebras (see [Nik96]). Nikulin and Gritsenko constructed families of examples of such algebras and gave a partial classification of them in rank 3 (see GN02] and the references therein). A complete classification of the rank 3 root systems with a timelike Weyl vector that 
satisfy the Gritsenko-Nikulin conditions was obtained recently by Allcock All15. The special features of the Gritsenko-Nikulin root systems make the classification problem more accessible, while their relation to Lie theory makes it particularly interesting.

Complex reflection groups. A complex reflection is an automorphism of a finitedimensional complex vector space that fixes a complex hyperplane. In contrast with the real case, complex reflections do not necessarily have order 2. Finite linear groups generated by complex reflections were classified by Shephard and Todd ST54. They are distinguished from all other finite linear groups by the property that their algebras of invariants are free. Realizing Hermitian symmetric spaces as bounded symmetric domains in a complex vector space allows us to define complex reflections in such spaces. It is well known that the only bounded symmetric domains which admit totally geodesic complex hypersurfaces are the complex hyperbolic spaces $\mathbb{C} \mathbb{H}^{n}$ (corresponding to the groups $\mathrm{U}(n, 1)$ ) and the domains of type IV (of the groups $\mathrm{O}(n, 2)$ ). It follows that only these spaces admit complex reflections.

The study of lattices in the complex hyperbolic space $\mathbb{C H}^{n}$ goes back to the nineteenth century. In the 1883 paper Pic83, Picard investigated the lattices $\mathrm{SU}\left(2,1 ; O_{d}\right)$, where $O_{d}$ is the ring of integers of the imaginary quadratic field $\mathbb{Q}(\sqrt{-d})$, acting on $\mathbb{C H}^{2}$. These groups are called the Picard modular groups. In a recent article PW13, Paupert and Will showed that for $d=1,2,3,7,11$ the Picard modular groups are up to a finite index generated by real reflections (i.e., antiholomorphic involutions that have a real totally geodesic plane fixed). It is not known which of the Picard groups are complex reflective. In an influential paper [Mos80, Mostow constructed Dirichlet fundamental domains for certain groups generated by complex reflections in $\mathbb{C} \mathbb{H}^{2}$, both arithmetic and non-arithmetic. The only other complex hyperbolic space in which a non-arithmetic lattice is known is $\mathbb{C H}^{3}$ DM86. Higher-dimensional examples of arithmetic complex hyperbolic reflection groups were constructed by Allcock in All00b, All00a]. His method is close to the constructions in the real hyperbolic spaces considered in this survey. In All00b, Allcock obtained examples of lattices generated by complex reflections in $\mathbb{C} \mathbb{H}^{5}, \mathbb{C H}{ }^{9}$, and $\mathbb{C} \mathbb{H}^{13}$ by considering the automorphism groups of Lorentzian lattices over the Eisenstein integers $O_{3}$. In [All00a], he used a related construction to give several other examples of lattices, including examples in $\mathbb{C H}^{4}$ and $\mathbb{C H}^{7}$ which do not appear on the list of Deligne and Mostow [DM86]. The Allcock group in a record high dimension 13 is again related to the Leech lattice. Some interesting examples of complex hyperbolic reflection groups are considered in [Der06], [Sto14], and DPP15]. A survey of the known constructions of complex hyperbolic lattices by Parker can be found in Par09].

Currently available results about complex hyperbolic reflection groups and lattices essentially fall into several types of the known constructions. There are no general finiteness theorems or classification attempts. Most of the questions that were discussed in this survey about real hyperbolic reflection groups can also be asked in the complex case, but here the results will turn into conjectures or open problems. It is not even clear, for instance, if we should expect that the dimension of arithmetic reflection complex hyperbolic lattices is bounded from above.

The case of domains $\mathbb{D}_{n}$ of type IV is different. Here the group $\mathrm{O}(n, 2)$ has real rank 2 , so we can obtain many examples of lattices generated by complex reflections 
for any $n$ using the construction for higher rank groups described in the beginning of this section. The natural question that comes out again is how to narrow this class. Vinberg suggested using as a criterion freeness of the algebra of automorphic forms on $\mathbb{D}_{n}$ invariant under $\Gamma$, thus generalizing the free polynomial invariant algebras of the finite complex reflection groups. There are some particularly interesting examples of lattices generated by complex reflections arising in this way. Igusa's paper Igu62 provides such an example for $n=3$. His results were largely extended by Vinberg in Vin10, whose construction provides this type of examples of arithmetic complex reflection groups in the domains of type IV for $n=4,5,6$, 7 .

In view of Borcherds' work Bor00] discussed in Section 8, one can ask if arithmetic complex reflection groups can be related to modular forms in a similar way as is done for the real reflective lattices. There are some known examples of this kind (see, e.g., All00b] but the general picture remains mysterious.

\section{ACKNOWLEDGMENTS}

The author is grateful to Ernest Borisovich Vinberg and Daniel Allcock for their comments and corrections, which helped to significantly improve the quality of this paper. The author would like to thank Alice Mark for sending me the preliminary version of her thesis and Igor Dolgachev for sending me his lecture notes. Comments from Richard Borcherds, Benjamin Linowitz, John Parker, John Ratcliffe, and the referee on the preliminary version of the paper were very helpful. This work was partially supported by the $\mathrm{CNPq}$ and FAPERJ research grants.

\section{ABOut THE AUTHOR}

Mikhail Belolipetsky is a researcher at IMPA, Rio de Janeiro, Brazil. He received his Ph.D. at Novosibirsk State University, Russia, in 2000, and he has held positions at Sobolev Institute of Mathematics (Novosibirsk, Russia), Max Plank Institute for Mathematics (Bonn, Germany), Hebrew University (Jerusalem, Israel) and Durham University (United Kingdom).

\section{REFERENCES}

[ABSW08] Ian Agol, Mikhail Belolipetsky, Peter Storm, and Kevin Whyte, Finiteness of arithmetic hyperbolic reflection groups, Groups Geom. Dyn. 2 (2008), no. 4, 481-498, DOI 10.4171/GGD/47. MR2442945 (2009m:20054)

[ACMR09] Omar Antolín-Camarena, Gregory R. Maloney, and Roland K. W. Roeder, Computing arithmetic invariants for hyperbolic reflection groups, Complex dynamics, A K Peters, Wellesley, MA, 2009, pp. 597-631, DOI 10.1201/b10617-22. MR2508271(2010h:20115)

[Ago06] Ian Agol, Finiteness of arithmetic Kleinian reflection groups, International Congress of Mathematicians. Vol. II, Eur. Math. Soc., Zürich, 2006, pp. 951-960. MR2275630 (2008e:30053)

[All99] Daniel Allcock, Reflection groups on the octave hyperbolic plane, J. Algebra 213 (1999), no. 2, 467-498, DOI 10.1006/jabr.1998.7671. MR.1673465 (2000e:17028)

[All00a] Daniel Allcock, New complex- and quaternion-hyperbolic reflection groups, Duke Math. J. 103 (2000), no. 2, 303-333, DOI 10.1215/S0012-7094-00-10326-2. MR:1760630(2001f:11105)

[All00b] Daniel Allcock, The Leech lattice and complex hyperbolic reflections, Invent. Math. 140 (2000), no. 2, 283-301, DOI 10.1007/s002220050363. MR1756997(2002b:11091)

[All06] Daniel Allcock, Infinitely many hyperbolic Coxeter groups through dimension 19, Geom. Topol. 10 (2006), 737-758 (electronic), DOI 10.2140/gt.2006.10.737. MR2240904(2007f:20067) 
[All12] Daniel Allcock, The reflective Lorentzian lattices of rank 3, Mem. Amer. Math. Soc. 220 (2012), no. 1033, x+108, DOI 10.1090/S0065-9266-2012-00648-4. MR3025355

[All13] Daniel Allcock, Reflection centralizers in Coxeter groups, Transform. Groups 18 (2013), no. 3, 599-613, DOI 10.1007/s00031-013-9236-7. MR3084328

[All15] Daniel Allcock, Root systems for Lorentzian Kac-Moody algebras in rank 3, Bull. Lond. Math. Soc. 47 (2015), no. 2, 325-342, DOI 10.1112/blms/bdv007. MR.3335126

[And70a] E. M. Andreev, Convex polyhedra in Lobačevskǐ̌ spaces (Russian), Mat. Sb. (N.S.) 81 (123) (1970), 445-478. MR0259734 (41 \#4367)

[And70b] E. M. Andreev, Convex polyhedra of finite volume in Lobačevskǐ space (Russian), Mat. Sb. (N.S.) 83 (125) (1970), 256-260. MR0273510 (42 \#8388)

[AVS93] D. V. Alekseevskij, E. B. Vinberg, and A. S. Solodovnikov, Geometry of spaces of constant curvature, Geometry, II, Encyclopaedia Math. Sci., vol. 29, Springer, Berlin, 1993, pp. 1-138, DOI 10.1007/978-3-662-02901-5_1. MR1254932 (95b:53042)

[Bar03] Alexander Graham Barnard, The singular theta correspondence, Lorentzian lattices and Borcherds-Kac-Moody algebras, ProQuest LLC, Ann Arbor, MI, 2003. Thesis (Ph.D.)-University of California, Berkeley. MR.2705173

[BE12] Mikhail Belolipetsky and Vincent Emery, On volumes of arithmetic quotients of $\mathrm{PO}(n, 1)^{\circ}, n$ odd, Proc. Lond. Math. Soc. (3) 105 (2012), no. 3, 541-570, DOI 10.1112/plms/pds009. MR2974199

[Bel04] Mikhail Belolipetsky, On volumes of arithmetic quotients of $\mathrm{SO}(1, n)$, Ann. Sc. Norm. Super. Pisa Cl. Sci. (5) 3 (2004), no. 4, 749-770. MR2124587 (2005k:11080)

[Bel07] Mikhail Belolipetsky, Addendum to "On volumes of arithmetic quotients of $\operatorname{SO}(1, n)$ ", Ann. Sc. Norm. Super. Pisa Cl. Sci. (5) 6 (2007), no. 2, 263-268. MR2124587 (2005k:11080)

[Bel09] Mikhail Belolipetsky, On fields of definition of arithmetic Kleinian reflection groups, Proc. Amer. Math. Soc. 137 (2009), no. 3, 1035-1038, DOI 10.1090/S0002-9939-0809590-7. MR2457444 (2009h:20041)

[Bel11] Mikhail Belolipetsky, Finiteness theorems for congruence reflection groups, Transform. Groups 16 (2011), no. 4, 939-954, DOI 10.1007/s00031-011-9156-3. MR.2852486 (2012k:20070)

[Bel14] Mikhail Belolipetsky, "Hyperbolic orbifolds of small volume". In International Congress of Mathematicians. Vol. II (Seoul 2014), pages 837-851. Kyung Moon SA, 2014.

[BHC62] Armand Borel and Harish-Chandra, Arithmetic subgroups of algebraic groups, Ann. of Math. (2) 75 (1962), 485-535. MR0147566 (26 \#5081)

[Bia91] Luigi Bianchi, Geometrische Darstellung der Gruppen linearer Substitutionen mit ganzen complexen Coefficienten nebst Anwendungen auf die Zahlentheorie (German), Math. Ann. 38 (1891), no. 3, 313-333, DOI 10.1007/BF01199425. MR.1510677

[BJS88] M. Bożejko, T. Januszkiewicz, and R. J. Spatzier, Infinite Coxeter groups do not have Kazhdan's property, J. Operator Theory 19 (1988), no. 1, 63-67. MR.950825 (89i:22025)

[BL14] Mikhail Belolipetsky and Benjamin Linowitz, On fields of definition of arithmetic Kleinian reflection groups II, Int. Math. Res. Not. IMRN 9 (2014), 2559-2571. MR3207375

[BLS92] M. Burger, J.-S. Li, and P. Sarnak, Ramanujan duals and automorphic spectrum, Bull. Amer. Math. Soc. (N.S.) 26 (1992), no. 2, 253-257, DOI 10.1090/S0273-0979-199200267-7. MR1118700 (92h:22023)

[BM13] Mikhail Belolipetsky and John Mcleod, Reflective and quasi-reflective Bianchi groups, Transform. Groups 18 (2013), no. 4, 971-994, DOI 10.1007/s00031-013-9245-6. MR.3127984

[Bor81] A. Borel, Commensurability classes and volumes of hyperbolic 3-manifolds, Ann. Scuola Norm. Sup. Pisa Cl. Sci. (4) 8 (1981), no. 1, 1-33. MR616899 (82j:22008)

[Bor87] Richard Borcherds, Automorphism groups of Lorentzian lattices, J. Algebra 111 (1987), no. 1, 133-153, DOI 10.1016/0021-8693(87)90245-6. MR913200 (89b:20018)

[Bor90] Richard E. Borcherds, Lattices like the Leech lattice, J. Algebra 130 (1990), no. 1, 219-234, DOI 10.1016/0021-8693(90)90110-A. MR1045746(91f:11046)

[Bor98] Richard E. Borcherds, Automorphic forms with singularities on Grassmannians, Invent. Math. 132 (1998), no. 3, 491-562, DOI 10.1007/s002220050232. MR.1625724 (99c:11049) 
[Bor00] Richard E. Borcherds, Reflection groups of Lorentzian lattices, Duke Math. J. 104 (2000), no. 2, 319-366, DOI 10.1215/S0012-7094-00-10424-3. MR.1773561 (2001h:11086)

[Bou68] N. Bourbaki, Éléments de mathématique. Fasc. XXXIV. Groupes et algèbres de Lie. Chapitre IV: Groupes de Coxeter et systèmes de Tits. Chapitre V: Groupes engendrés par des réflexions. Chapitre VI: systèmes de racines (French), Actualités Scientifiques et Industrielles, No. 1337, Hermann, Paris, 1968. MR0240238 (39 \#1590)

[BS91] M. Burger and P. Sarnak, Ramanujan duals. II, Invent. Math. 106 (1991), no. 1, 1-11, DOI 10.1007/BF01243900. MR1123369 (92m:22005)

[Bug84] V. O. Bugaenko, Groups of automorphisms of unimodular hyperbolic quadratic forms over the ring $\mathbf{Z}[(\sqrt{5}+1) / 2]$ (Russian), Vestnik Moskov. Univ. Ser. I Mat. Mekh. 5 (1984), 6-12. MR764026 (86d:11030)

[Bug90] V. O. Bugaenko, On reflective unimodular hyperbolic quadratic forms, Selecta Math. Soviet. 9 (1990), no. 3, 263-271. Selected translations. MR.1074386 (92g:11033)

[Bug92] V. O. Bugaenko, Arithmetic crystallographic groups generated by reflections, and reflective hyperbolic lattices, Lie groups, their discrete subgroups, and invariant theory, Adv. Soviet Math., vol. 8, Amer. Math. Soc., Providence, RI, 1992, pp. 33-55. MR.1155663 (93g:20094)

[CF86] Ted Chinburg and Eduardo Friedman, The smallest arithmetic hyperbolic threeorbifold, Invent. Math. 86 (1986), no. 3, 507-527, DOI 10.1007/BF01389265. MR860679 (88a:22022)

[Con83] J. H. Conway, The automorphism group of the 26-dimensional even unimodular Lorentzian lattice, J. Algebra 80 (1983), no. 1, 159-163, DOI 10.1016/00218693(83)90025-X. MR690711(85k:11030)

[Cox34] H. S. M. Coxeter, Discrete groups generated by reflections, Ann. of Math. (2) 35 (1934), no. 3, 588-621, DOI 10.2307/1968753. MR1503182

[CS99] J. H. Conway and N. J. A. Sloane, Sphere packings, lattices and groups, 3rd ed., Grundlehren der Mathematischen Wissenschaften [Fundamental Principles of Mathematical Sciences], vol. 290, Springer-Verlag, New York, 1999. With additional contributions by E. Bannai, R. E. Borcherds, J. Leech, S. P. Norton, A. M. Odlyzko, R. A. Parker, L. Queen and B. B. Venkov. MR 1662447 (2000b:11077)

[CW50] H. S. M. Coxeter and G. J. Whitrow, World-structure and non-Euclidean honeycombs, Proc. Roy. Soc. London. Ser. A. 201 (1950), 417-437. MR0041576 (12,866e)

[Der06] Martin Deraux, Deforming the $\mathbb{R}$-Fuchsian (4,4,4)-triangle group into a lattice, Topology 45 (2006), no. 6, 989-1020, DOI 10.1016/j.top.2006.06.005. MR2263221 (2007m:32015)

[DK03] I. Dolgachev and S. Kondō, A supersingular K3 surface in characteristic 2 and the Leech lattice, Int. Math. Res. Not. 1 (2003), 1-23, DOI 10.1155/S1073792803202038. MR1935564(2003i:14051)

[DM86] P. Deligne and G. D. Mostow, Monodromy of hypergeometric functions and nonlattice integral monodromy, Inst. Hautes Études Sci. Publ. Math. 63 (1986), 5-89. MR.849651 (88a:22023a)

[Dol08] Igor V. Dolgachev, Reflection groups in algebraic geometry, Bull. Amer. Math. Soc. (N.S.) 45 (2008), no. 1, 1-60, DOI 10.1090/S0273-0979-07-01190-1. MR.2358376 (2009h:14001)

[Dol15] I. Dolgachev, Hyperbolic geometry and algebraic geometry. Seoul-Austin Lecture Notes, available at http://www.math.lsa.umich.edu/ idolga/lecturenotes.html, 2015.

[DPP15] Martin Deraux, John R. Parker, and Julien Paupert, New non-arithmetic complex hyperbolic lattices. 2015. to appear.

[Duf10] Guillaume Dufour, Notes on right-angled Coxeter polyhedra in hyperbolic spaces, Geom. Dedicata 147 (2010), 277-282, DOI 10.1007/s10711-009-9454-2. MR 2660580 (2011g:20056)

[Dyc82] Walther Dyck, Gruppentheoretische Studien (German), Math. Ann. 20 (1882), no. 1, 1-44, DOI 10.1007/BF01443322. MR 1510147

[Ess96] Frank Esselmann, Über die maximale Dimension von Lorentz-Gittern mit coendlicher Spiegelungsgruppe (German, with English summary), J. Number Theory 61 (1996), no. 1, 103-144, DOI 10.1006/jnth.1996.0141. MR1418323(97g:11073) 
[FMS14] Elena Fuchs, Chen Meiri, and Peter Sarnak, Hyperbolic monodromy groups for the hypergeometric equation and Cartan involutions, J. Eur. Math. Soc. (JEMS) 16 (2014), no. 8, 1617-1671, DOI 10.4171/JEMS/471. MR3262453

[Fri91] Robert Fricke, Ueber eine besondere Classe discontinuirlicher Gruppen reeller linearer Substitutionen (German), Math. Ann. 38 (1891), no. 1, 50-81, DOI 10.1007/BF01212693. MR1510663

[FT14] Anna Felikson and Pavel Tumarkin, Essential hyperbolic Coxeter polytopes, Israel J. Math. 199 (2014), no. 1, 113-161, DOI 10.1007/s11856-013-0046-3. MR.3219531

[GC] The Geometry Center, University of Minnesota. Current homepage address http://www.geom. uiuc.edu.

[GM09] Frederick W. Gehring and Gaven J. Martin, Minimal co-volume hyperbolic lattices. I. The spherical points of a Kleinian group, Ann. of Math. (2) 170 (2009), no. 1, 123-161, DOI 10.4007/annals.2009.170.123. MR2521113(2010h:57029)

[GN02] V. A. Gritsenko and V. V. Nikulin, On the classification of Lorentzian Kac-Moody algebras (Russian, with Russian summary), Uspekhi Mat. Nauk 57 (2002), no. 5(347), 79-138, DOI 10.1070/RM2002v057n05ABEH000553; English transl., Russian Math. Surveys 57 (2002), no. 5, 921-979. MR1992083 (2004f:17034)

[Her70] Joseph Hersch, Quatre propriétés isopérimétriques de membranes sphériques homogènes (French), C. R. Acad. Sci. Paris Sér. A-B 270 (1970), A1645-A1648. MR0292357 (45 \#1444)

[HM96] Jeffrey A. Harvey and Gregory Moore, Algebras, BPS states, and strings, Nuclear Phys. B 463 (1996), no. 2-3, 315-368, DOI 10.1016/0550-3213(95)00605-2. MR:1393643(97h:81163)

[Igu62] Jun-ichi Igusa, On Siegel modular forms of genus two, Amer. J. Math. 84 (1962), 175-200. MR0141643 (25 \#5040)

$\left[J^{+}{ }^{15}\right.$ ] E. Jespers, S. O. Juriaans, A. Kiefer, A. de A. e Silva, and A. C. Souza Filho, From the Poincaré theorem to generators of the unit group of integral group rings of finite groups, Math. Comp. 84 (2015), no. 293, 1489-1520, DOI 10.1090/S0025-5718-201402865-2. MR 3315518

[JKRT02] N. W. Johnson, R. Kellerhals, J. G. Ratcliffe, and S. T. Tschantz, Commensurability classes of hyperbolic Coxeter groups, Linear Algebra Appl. 345 (2002), 119-147, DOI 10.1016/S0024-3795(01)00477-3. MR:1883270 (2002m:20062)

[Kaz67] D. A. Každan, On the connection of the dual space of a group with the structure of its closed subgroups (Russian), Funkcional. Anal. i Priložen. 1 (1967), 71-74. MR0209390 (35 \#288)

[Kle79] Felix Klein, Ueber die Transformation siebenter Ordnung der elliptischen Functionen (German), Math. Ann. 14 (1878), no. 3, 428-471, DOI 10.1007/BF01677143. MR 1509988

[KM68] D. A. Každan and G. A. Margulis, A proof of Selberg's hypothesis (Russian), Mat. Sb. (N.S.) 75 (117) (1968), 163-168. MR0223487 (36 \#6535)

[Lak12a] Grant Lakeland, Arithmetic reflection groups and congruence subgroups. PhD thesis, University of Texas at Austin, 2012.

[Lak12b] Grant S. Lakeland, Dirichlet-Ford domains and arithmetic reflection groups, Pacific J. Math. 255 (2012), no. 2, 417-437, DOI 10.2140/pjm.2012.255.417. MR2928559

[Lan50] Folke Lannér, On complexes with transitive groups of automorphisms, Comm. Sém., Math. Univ. Lund [Medd. Lunds Univ. Mat. Sem.] 11 (1950), 71. MR0042129(13,58c)

[Lev99] Silvio Levy, editor, The eightfold way: The beauty of Klein's quartic curve, volume 35 of Mathematical Sciences Research Institute Publications. Cambridge University Press, Cambridge, 1999.

[LMR06] D. D. Long, C. Maclachlan, and A. W. Reid, Arithmetic Fuchsian groups of genus zero, Pure Appl. Math. Q. 2 (2006), no. 2, 569-599, DOI 10.4310/PAMQ.2006.v2.n2.a9. MR2251482 (2007f:20092)

[LRS99] Wenzhi Luo, Zeév Rudnick, and Peter Sarnak, "On the generalized Ramanujan conjecture for GL(n)". In Automorphic forms, automorphic representations, and arithmetic (Fort Worth, TX, 1996), volume 66 of Proc. Sympos. Pure Math., pages 301-310. Amer. Math. Soc., Providence, RI, 1999. 
[LY82] Peter Li and Shing Tung Yau, A new conformal invariant and its applications to the Willmore conjecture and the first eigenvalue of compact surfaces, Invent. Math. 69 (1982), no. 2, 269-291, DOI 10.1007/BF01399507. MR674407 (84f:53049)

[Ma15] Shouhei Ma, Finiteness of 2-reflective lattices of signature $(2, n)$. Preprint arXiv: 1409.2969 [math.AG], 2015.

[Mac11] C. Maclachlan, Bounds for discrete hyperbolic arithmetic reflection groups in dimension 2, Bull. Lond. Math. Soc. 43 (2011), no. 1, 111-123, DOI 10.1112/blms/bdq085. MR2765555 (2012c:11093)

[Mar91] G. A. Margulis, Discrete subgroups of semisimple Lie groups, Ergebnisse der Mathematik und ihrer Grenzgebiete (3) [Results in Mathematics and Related Areas (3)], vol. 17, Springer-Verlag, Berlin, 1991. MR.1090825 (92h:22021)

[Mar15a] Alice Mark, Reflection groups of the quadratic form $-p x_{0}^{2}+x_{1}^{2}+\cdots+x_{n}^{2}$ with p prime, Publ. Mat. 59 (2015), no. 2, 353-372. MR3374612

[Mar15b] Alice Mark, The classification of rank 3 reflective hyperbolic lattices over $\mathbb{Z}(\sqrt{2})$. University of Texas at Austin, PhD thesis, May 2015. http://hdl.handle.net/2152/ 31507

[Mcl11] John Mcleod, Hyperbolic reflection groups associated to the quadratic forms $-3 x_{0}^{2}+$ $x_{1}^{2}+\cdots+x_{n}^{2}$, Geom. Dedicata 152 (2011), 1-16, DOI 10.1007/s10711-010-9543-2. MR2795233 (2012c:20107)

[Mcl13] John Angus McLeod, Arithmetic Hyperbolic Reflection Groups, ProQuest LLC, Ann Arbor, MI, 2013. Thesis (Ph.D.)-University of Durham (United Kingdom). MR3389315

[Mei14] Chen Meiri, On the non-amenability of the reflective quotient I: The rational case. Preprint arXiv:1401.7708 [math.GR], 2014.

[Miy89] Toshitsune Miyake, Modular forms, Reprint of the first 1989 English edition, Springer Monographs in Mathematics, Springer-Verlag, Berlin, 2006. Translated from the 1976 Japanese original by Yoshitaka Maeda. MR2194815 (2006g:11084)

[MM12] T. H. Marshall and G. J. Martin, Minimal co-volume hyperbolic lattices, II: Simple torsion in a Kleinian group, Ann. of Math. (2) 176 (2012), no. 1, 261-301, DOI 10.4007/annals.2012.176.1.4. MR2925384

[Mos80] G. D. Mostow, On a remarkable class of polyhedra in complex hyperbolic space, Pacific J. Math. 86 (1980), no. 1, 171-276. MR586876 (82a:22011)

[MOv12] G. D. Mostow, Actions on $\mathbb{H}^{n}$ generated by torsion elements. MathOverflow Question http://mathoverflow.net/q/102644, 2012.

[MR03] Colin Maclachlan and Alan W. Reid, The arithmetic of hyperbolic 3-manifolds, Graduate Texts in Mathematics, vol. 219, Springer-Verlag, New York, 2003. MR.1937957 (2004i:57021)

[Nik79] V. V. Nikulin, Quotient-groups of groups of automorphisms of hyperbolic forms of subgroups generated by 2-reflections (Russian), Dokl. Akad. Nauk SSSR 248 (1979), no. 6, 1307-1309. MR556762 (81d:10011)

[Nik80] V. V. Nikulin, On the arithmetic groups generated by reflections in Lobačevskiน spaces (Russian), Izv. Akad. Nauk SSSR Ser. Mat. 44 (1980), no. 3, 637-669, 719-720. MR.582161 (82m:57029)

[Nik81a] V. V. Nikulin, On the classification of arithmetic groups generated by reflections in Lobachevskiて spaces (Russian), Izv. Akad. Nauk SSSR Ser. Mat. 45 (1981), no. 1, 113-142, 240. MR607579 (83b:57025)

[Nik81b] V. V. Nikulin, Quotient-groups of groups of automorphisms of hyperbolic forms by subgroups generated by 2-reflections. Algebro-geometric applications (Russian), Current problems in mathematics, Vol. 18, Akad. Nauk SSSR, Vsesoyuz. Inst. Nauchn. i Tekhn. Informatsii, Moscow, 1981, pp. 3-114. MR633160 (83c:10030)

[Nik84] V. V. Nikulin, K3 surfaces with a finite group of automorphisms and a Picard group of rank three (Russian), Trudy Mat. Inst. Steklov. 165 (1984), 119-142. Algebraic geometry and its applications. MR.752938 (86e:14018)

[Nik87] V. V. Nikulin, Discrete reflection groups in Lobachevsky spaces and algebraic surfaces, Proceedings of the International Congress of Mathematicians, Vol. 1, 2 (Berkeley, Calif., 1986), Amer. Math. Soc., Providence, RI, 1987, pp. 654-671. MR.934268 (89d:11032) 
[Nik96] V. V. Nikulin, Reflection groups in Lobachevski spaces and an identity for the denominator of Lorentzian Kac-Moody algebras (Russian, with Russian summary), Izv. Ross. Akad. Nauk Ser. Mat. 60 (1996), no. 2, 73-106, DOI 10.1070/IM1996v060n02ABEH000072; English transl., Izv. Math. 60 (1996), no. 2, 305-334. MR.1399419 (97f:17026)

[Nik00] V. V. Nikulin, On the classification of hyperbolic root systems of rank three (Russian, with English and Russian summaries), Tr. Mat. Inst. Steklova 230 (2000), 256; English transl., Proc. Steklov Inst. Math. 3 (230) (2000), 1-241. MR1802343 (2001m:17034)

[Nik07] V. V. Nikulin, Finiteness of the number of arithmetic groups generated by reflections in Lobachevskiu spaces (Russian, with Russian summary), Izv. Ross. Akad. Nauk Ser. Mat. 71 (2007), no. 1, 55-60, DOI 10.1070/IM2007v071n01ABEH002349; English transl., Izv. Math. 71 (2007), no. 1, 53-56. MR2477273 (2010b:20068)

[Nik09] Viacheslav V. Nikulin, On ground fields of arithmetic hyperbolic reflection groups, Groups and symmetries, CRM Proc. Lecture Notes, vol. 47, Amer. Math. Soc., Providence, RI, 2009, pp. 299-326. MR2500568 (2010h:11057)

[Nik11] V. V. Nikulin, The transition constant for arithmetic hyperbolic reflection groups (Russian, with Russian summary), Izv. Ross. Akad. Nauk Ser. Mat. 75 (2011), no. 5, 103-138, DOI 10.1070/IM2011v075n05ABEH002561; English transl., Izv. Math. 75 (2011), no. 5, 971-1005. MR2884665(2012m:11055)

[Oni87] A. L. Onishchik, editor, Voprosy Teorii Grupp i Gomologicheskoi Algebry. Yaroslavl, 1987. English translation: Selecta Math. Soviet., Vol. 9, No. 4 (1990).

[Par09] John R. Parker, Complex hyperbolic lattices, Discrete groups and geometric structures, Contemp. Math., vol. 501, Amer. Math. Soc., Providence, RI, 2009, pp. 1-42, DOI 10.1090/conm/501/09838. MR2581913(2011g:22018)

[Pic83] Emile Picard, Sur des fonctions de deux variables indépendantes analogues aux fonctions modulaires (French), Acta Math. 2 (1883), no. 1, 114-135, DOI 10.1007/BF02415213. MR 1554595

[Poi82] H. Poincaré, Théorie des groupes fuchsiens (French), Acta Math. 1 (1882), no. 1, 1-76, DOI 10.1007/BF02391835. MR 1554574

[PR94] Vladimir Platonov and Andrei Rapinchuk, Algebraic groups and number theory, Pure and Applied Mathematics, vol. 139, Academic Press, Inc., Boston, MA, 1994. Translated from the 1991 Russian original by Rachel Rowen. MR1278263 (95b:11039)

[Pra89] Gopal Prasad, Volumes of S-arithmetic quotients of semi-simple groups, Inst. Hautes Études Sci. Publ. Math. 69 (1989), 91-117. With an appendix by Moshe Jarden and the author. MR1019962 (91c:22023)

[Pro86] M. N. Prokhorov, Absence of discrete groups of reflections with a noncompact fundamental polyhedron of finite volume in a Lobachevskǐ space of high dimension (Russian), Izv. Akad. Nauk SSSR Ser. Mat. 50 (1986), no. 2, 413-424. MR842588 (87k:22016)

[PV05] Leonid Potyagailo and Ernest Vinberg, On right-angled reflection groups in hyperbolic spaces, Comment. Math. Helv. 80 (2005), no. 1, 63-73, DOI 10.4171/CMH/4. MR2130566 (2006a:20076)

[PW13] Julien Paupert and Pierre Will, Real reflections, commutators and cross-ratios in complex hyperbolic space. Preprint arXiv:1312.3173 [math.GT], 2013.

[Rat06] John G. Ratcliffe, Foundations of hyperbolic manifolds, 2nd ed., Graduate Texts in Mathematics, vol. 149, Springer, New York, 2006. MR2249478 (2007d:57029)

[Roe07] Roland K. W. Roeder, Constructing hyperbolic polyhedra using Newton's method, Experiment. Math. 16 (2007), no. 4, 463-492. MR2378487(2009h:65029)

[Ruz89] O. P. Ruzmanov, Examples of nonarithmetic crystallographic Coxeter groups in $n$ dimensional Lobachevskiน space when $6 \leq n \leq 10$ (Russian), Problems in group theory and in homological algebra (Russian), Yaroslav. Gos. Univ., Yaroslavl', 1989, pp. 138142. MR 1068774 (91m:20070)

[Ruz90] O. P. Ruzmanov, Subgroups of reflections in Bianchi groups (Russian), Uspekhi Mat. Nauk 45 (1990), no. 1(271), 189-190, DOI 10.1070/RM1990v045n01ABEH002323; English transl., Russian Math. Surveys 45 (1990), no. 1, 227-228. MR 1050942 (91g:11043) 
[Sar14] Peter Sarnak, Notes on thin matrix groups, Thin groups and superstrong approximation, Math. Sci. Res. Inst. Publ., vol. 61, Cambridge Univ. Press, Cambridge, 2014, pp. 343-362. MR3220897

[SB96] Rudolf Scharlau and Britta Blaschke, Reflective integral lattices, J. Algebra 181 (1996), no. 3, 934-961, DOI 10.1006/jabr.1996.0155. MR1386588 (97c:11072)

[Sch89] Rudolf Scharlau, On the classification of arithmetic reflection groups on hyperbolic 3-space. Preprint, Bielefeld, 1989.

[Sha90] M. K. Shaiheev, Reflective subgroups in Bianchi groups, Selecta Math. Soviet. 9 (1990), no. 4, 315-322. Selected translations. MR.1078260 (91j:20118)

[Shv90] O. V. Shvartsman, Reflective subgroups of Bianchi groups, Selecta Math. Soviet. 9 (1990), no. 4, 323-329. Selected translations. MR.1078261 (91j:20119)

[ST54] G. C. Shephard and J. A. Todd, Finite unitary reflection groups, Canadian J. Math. 6 (1954), 274-304. MR0059914 (15,600b)

[Sto14] Matthew Stover, Hurwitz ball quotients, Math. Z. 278 (2014), no. 1-2, 75-91, DOI 10.1007/s00209-014-1306-6. MR.3267570

[SW92] Rudolf Scharlau and Claudia Walhorn, Integral lattices and hyperbolic reflection groups, Astérisque 209 (1992), 15-16, 279-291. Journées Arithmétiques, 1991 (Geneva). MR 1211022 (94j:11057)

[Sze54] G. Szegö, Inequalities for certain eigenvalues of a membrane of given area, J. Rational Mech. Anal. 3 (1954), 343-356. MR0061749 (15,877c)

[Thu97] William P. Thurston, Three-dimensional geometry and topology. Vol. 1, volume 35 of Princeton Mathematical Series. Princeton University Press, Princeton, NJ, 1997. Edited by Silvio Levy.

[Tit66] J. Tits, Classification of algebraic semisimple groups, Algebraic Groups and Discontinuous Subgroups (Proc. Sympos. Pure Math., Boulder, Colo., 1965), Amer. Math. Soc., Providence, R.I., 1966, 1966, pp. 33-62. MR0224710 (37 \#309)

[Tit72] J. Tits, Free subgroups in linear groups, J. Algebra 20 (1972), 250-270. MR.0286898 (44 \#4105)

[Tur] Ivica Turkalj, Totally-reflective genera of integral lattices. Preprint arXiv:1503. 04428v2 [math.NT].

[VGS88] È. B. Vinberg, V. V. Gorbatsevich, and O. V. Shvartsman, Discrete subgroups of Lie groups (Russian), Current problems in mathematics. Fundamental directions, Vol. 21 (Russian), Itogi Nauki i Tekhniki, Akad. Nauk SSSR, Vsesoyuz. Inst. Nauchn. i Tekhn. Inform., Moscow, 1988, pp. 5-120, 215. MR968445 (90c:22036)

[Vig83] Marie-France Vignéras, Quelques remarques sur la conjecture $\lambda_{1} \geq \frac{1}{4}$ (French), Seminar on number theory, Paris 1981-82 (Paris, 1981/1982), Progr. Math., vol. 38, Birkhäuser Boston, Boston, MA, 1983, pp. 321-343. MR729180 (85c:11049)

[Vin67] È. B. Vinberg, Discrete groups generated by reflections in Lobačevskiน spaces (Russian), Mat. Sb. (N.S.) 72 (114) (1967), 471-488; correction, ibid. 73 (115) (1967), 303. MR0207853 (34 \#7667)

[Vin71] È. B. Vinberg, Rings of definition of dense subgroups of semisimple linear groups. (Russian), Izv. Akad. Nauk SSSR Ser. Mat. 35 (1971), 45-55. MR0279206(43 \#4929)

[Vin72] È. B. Vinberg, The groups of units of certain quadratic forms (Russian), Mat. Sb. (N.S.) 87(129) (1972), 18-36. MR0295193 (45 \#4261)

[Vin75] È. B. Vinberg, Some arithmetical discrete groups in Lobačevskiŭ spaces, Discrete subgroups of Lie groups and applications to moduli (Internat. Colloq., Bombay, 1973), Oxford Univ. Press, Bombay, 1975, pp. 323-348. MR0422505 (54 \#10492)

[Vin81] È. B. Vinberg, The nonexistence of crystallographic reflection groups in Lobachevskiน spaces of large dimension (Russian), Funktsional. Anal. i Prilozhen. 15 (1981), no. 2, 67-68. MR617472 (83d:51026)

[Vin84a] È. B. Vinberg, Absence of crystallographic groups of reflections in Lobachevskǐ spaces of large dimension (Russian), Trudy Moskov. Mat. Obshch. 47 (1984), 68-102, 246. MR774946 (86i:22020)

[Vin84b] È. B. Vinberg, Discrete reflection groups in Lobachevsky spaces, Proceedings of the International Congress of Mathematicians, Vol. 1, 2 (Warsaw, 1983), PWN, Warsaw, 1984, pp. 593-601. MR804716 (87h:22016) 
[Vin85] È. B. Vinberg, Hyperbolic groups of reflections (Russian), Uspekhi Mat. Nauk 40 (1985), no. 1(241), 29-66, 255. MR783604 (86m:53059)

[Vin90] È. B. Vinberg, Reflective subgroups in Bianchi groups, Selecta Math. Soviet. 9 (1990), no. 4, 309-314. Selected translations. MR1078259 (91j:20117)

[Vin07] È. B. Vinberg, Classification of 2-reflective hyperbolic lattices of rank 4 (Russian, with Russian summary), Tr. Mosk. Mat. Obs. 68 (2007), 44-76, DOI 10.1090/s0077-155407-00160-4; English transl., Trans. Moscow Math. Soc. (2007), 39-66. MR.2429266 (2009c:11099)

[Vin10] E. B. Vinberg, Some free algebras of automorphic forms on symmetric domains of type IV, Transform. Groups 15 (2010), no. 3, 701-741, DOI 10.1007/s00031-010-91074. MR2718942(2011k:32033)

[Vin14] È. B. Vinberg, Non-arithmetic hyperbolic reflection groups in higher dimensions. Univ. Bielefeld Preprint 14047, 2014.

[VK78] È. B. Vinberg and I. M. Kaplinskaja, The groups $O_{18,1}(Z)$ and $O_{19,1}(Z)$ (Russian), Dokl. Akad. Nauk SSSR 238 (1978), no. 6, 1273-1275. MR0476640(57 \#16199)

[Wa193] Claudia Walhorn, Arithmetische Spiegelungsgruppen auf dem 4-dimensionalen hyperbolischen Raum. PhD thesis, Univ. Bielefeld, 1993.

[Wan72] Hsien Chung Wang, Topics on totally discontinuous groups, Symmetric spaces (Short Courses, Washington Univ., St. Louis, Mo., 1969-1970), Dekker, New York, 1972, pp. 459-487. Pure and Appl. Math., Vol. 8. MR0414787 (54 \#2879)

[Wit15] Dave Witte Morris, Introduction to arithmetic groups, Deductive Press, Place of publication not identified, 2015. MR 3307755

[YY80] Paul C. Yang and Shing Tung Yau, Eigenvalues of the Laplacian of compact Riemann surfaces and minimal submanifolds, Ann. Scuola Norm. Sup. Pisa Cl. Sci. (4) 7 (1980), no. 1, 55-63. MR577325 (81m:58084)

[Zog91] P. Zograf, A spectral proof of Rademacher's conjecture for congruence subgroups of the modular group, J. Reine Angew. Math. 414 (1991), 113-116, DOI 10.1515/crll.1991.414.113. MR1092625(92d:11041)

IMPA, Estrada Dona Castorina, 110, 22460-320 Rio de Janeiro, BraziL

E-mail address: mbel@impa.br 